\title{
3. (1) Nivolumab plus ipilimumab versus sunitinib in first-line treatment for advanced renal cell carcinoma: extended follow-up of efficacy and safety results from a randomised, controlled, phase 3 trial
}

Robert J Motzer, Brian I Rini, David F McDermott, Osvaldo Arén Frontera, Hans J Hammers, Michael A Carducci, Pamela Salman, Bernard Escudier,
Benoit Beuselinck, Asim Amin, Camillo Porta, Saby George, Victoria Neiman, Sergio Bracarda, Scott S Tykodi, Philippe Barthélémy,
Raya Leibowitz-Amit, Elizabeth R Plimack, Sjoukje F Oosting, Bruce Redman, Bohuslav Melichar, Thomas Powles, Paul Nathan, Stéphane Oudard,
David Pook, Toni K Choueiri, Frede Donskov, Marc-Oliver Grimm, Howard Gurney, Daniel Y C Heng, Christian K Kollmannsberger, Michael R Harrison,
Yoshihiko Tomita, Ignacio Duran, Viktor Grünwald, M Brent McHenry, Sabeen Mekan, Nizar M Tannir, on behalf of the CheckMate 214 investigators*

Summary

Lancet Oncol 2019; 20: 1370-85 Published Online August 16, 2019 http://dx.doi.org/10.1016/ S1470-2045(19)30413-9 This online publication has been corrected. The corrected version first appeared at thelancet.com/oncology on August 21, 2019

See Comment page 1331 *Investigators listed in the appendix

Department of Medicine, Memorial Sloan Kettering

Cancer Center, New York, NY, USA (Prof RJ Motzer MD);

Department of Hematology and Medical Oncology, Cleveland Clinic Taussig Cancer Institute, Cleveland, $\mathrm{OH}$, USA (B I Rini MD); Department of Hematology and Medical Oncology, Beth Israel Deaconess Medical Center, Dana-Farber/Harvard Cancer

Center, Boston, MA, USA (D F McDermott MD); Department of Medical Oncology, Centro de Invetigación Clínica Bradford Hill, Santiago, Chile (O Arén Frontera MD); Department of Oncology and Urology, The Sidney Kimmel Comprehensive Cancer Center at Johns Hopkins, Baltimore, MD, USA (H) Hammers MD,

Prof M A (arducci MD);

Department of Medical Oncology, Fundación Arturo López Pérez, Santiago, Chile (P Salman MD); Department of Medical Oncology, Gustave Roussy, Villejuif, France (Prof B Escudier MD); Department of Oncology, University Hospitals Leuven, Leuven, Belgium

(B Beuselinck MD); Levine

Cancer Institute, Atrium

Background In the ongoing phase 3 CheckMate 214 trial, nivolumab plus ipilimumab showed superior efficacy over sunitinib in patients with previously untreated intermediate-risk or poor-risk advanced renal cell carcinoma, with a manageable safety profile. In this study, we aimed to assess efficacy and safety after extended follow-up to inform the long-term clinical benefit of nivolumab plus ipilimumab versus sunitinib in this setting.

Methods In the phase 3, randomised, controlled CheckMate 214 trial, patients aged 18 years and older with previously untreated, advanced, or metastatic histologically confirmed renal cell carcinoma with a clear-cell component were recruited from 175 hospitals and cancer centres in 28 countries. Patients were categorised by International Metastatic Renal Cell Carcinoma Database Consortium risk status into favourable-risk, intermediate-risk, and poor-risk subgroups and randomly assigned (1:1) to open-label nivolumab ( $3 \mathrm{mg} / \mathrm{kg}$ intravenously) plus ipilimumab (1 $\mathrm{mg} / \mathrm{kg}$ intravenously) every 3 weeks for four doses, followed by nivolumab ( $3 \mathrm{mg} / \mathrm{kg}$ intravenously) every 2 weeks; or sunitinib (50 mg orally) once daily for 4 weeks (6-week cycle). Randomisation was done through an interactive voice response system, with a block size of four and stratified by risk status and geographical region. The co-primary endpoints for the trial were overall survival, progression-free survival per independent radiology review committee (IRRC), and objective responses per IRRC in intermediate-risk or poor-risk patients. Secondary endpoints were overall survival, progression-free survival per IRRC, and objective responses per IRRC in the intention-to-treat population, and adverse events in all treated patients. In this Article, we report overall survival, investigator-assessed progression-free survival, investigatorassessed objective response, characterisation of response, and safety after extended follow-up. Efficacy outcomes were assessed in all randomly assigned patients; safety was assessed in all treated patients. This study is registered with ClinicalTrials.gov, number NCT02231749, and is ongoing but now closed to recruitment.

Findings Between Oct 16, 2014, and Feb 23, 2016, of 1390 patients screened, 1096 (79\%) eligible patients were randomly assigned to nivolumab plus ipilimumab or sunitinib $(550$ vs 546 in the intention-to-treat population; 425 vs 422 intermediate-risk or poor-risk patients, and 125 vs 124 favourable-risk patients). With extended follow-up (median follow-up 32.4 months [IQR 13.4-36.3]), in intermediate-risk or poor-risk patients, results for the three co-primary efficacy endpoints showed that nivolumab plus ipilimumab continued to be superior to sunitinib in terms of overall survival (median not reached [95\% CI 35.6-not estimable] vs $26 \cdot 6$ months [22.1-33.4]; hazard ratio [HR] 0.66 [95\% CI 0.54-0.80], p<0.0001), progression-free survival (median 8.2 months [95\% CI 6.9-10.0] vs $8 \cdot 3$ months [7.0-8.8]; HR 0.77 [95\% CI $0.65-0 \cdot 90], \mathrm{p}=0 \cdot 0014)$, and the proportion of patients achieving an objective response (178 [42\%] of 425 vs 124 [29\%] of 422; p=0.0001). Similarly, in intention-to-treat patients, nivolumab and ipilimumab showed improved efficacy compared with sunitinib in terms of overall survival (median not reached [95\% CI not estimable] vs 37.9 months [32.2-not estimable]; HR $0 \cdot 71$ [95\% CI 0.59-0 . 86], p=0 0003), progressionfree survival (median 9.7 months [95\% CI 8.1-11.1] vs 9.7 months [8.3-11.1]; HR 0.85 [95\% CI 0.73-0.98], $\mathrm{p}=0.027$ ), and the proportion of patients achieving an objective response (227 [41\%] of $550 \mathrm{vs} 186$ [34\%] of $546 \mathrm{p}=0.015)$. In all treated patients, the most common grade 3-4 treatment-related adverse events in the nivolumab and ipilimumab group were increased lipase (57 [10\%] of 547), increased amylase (31 [6\%]), and increased alanine aminotransferase (28 [5\%]), whereas in the sunitinib group they were hypertension (90 [17\%] of 535), fatigue (51 [10\%]), and palmarplantar erythrodysaesthesia (49 [9\%]). Eight deaths in the nivolumab plus ipilimumab group and four deaths in the sunitinib group were reported as treatment-related.

Interpretation The results suggest that the superior efficacy of nivolumab plus ipilimumab over sunitinib was maintained in intermediate-risk or poor-risk and intention-to-treat patients with extended follow-up, and show the 
long-term benefits of nivolumab plus ipilimumab in patients with previously untreated advanced renal cell carcinoma across all risk categories.

\section{Funding Bristol-Myers Squibb and ONO Pharmaceutical.}

\section{Copyright (C) 2019 Elsevier Ltd. All rights reserved.}

\section{Introduction}

The immunotherapy combination of nivolumab (a PD-1 immune checkpoint inhibitor antibody) plus

\section{Research in context}

\section{Evidence before this study}

We did a literature search on PubMed for published clinical trial reports, with no restrictions on article type or language, from database inception until May 13, 2019, using the terms "nivolumab," "nivolumab AND ipilimumab", "sunitinib", "renal cell carcinoma", "RCC", "kidney cancer", "advanced and metastatic RCC", "advanced and metastatic renal cell carcinoma", and "renal cell carcinoma", and with specific attention to randomised phase 3 trials with any of the following comparators: VEGF inhibitors or immuno-oncology therapeutics. The only randomised, open-label, phase 3 trial we found in first-line therapy was CheckMate 214, which compared nivolumab plus ipilimumab with sunitinib in patients with previously untreated, advanced, or metastatic renal cell carcinoma. Our search also showed several published randomised studies of relevance in patients with previously treated and untreated clear cell advanced renal cell carcinoma. Various targeted therapies, including antiangiogenic drugs targeting VEGF and its receptors and mTOR inhibitors, have improved outcomes for patients with advanced renal cell carcinoma in the past decade. Sunitinib (a VEGF tyrosine kinase inhibitor) has been considered a gold standard first-line treatment option for patients with advanced renal cell carcinoma. Yet, durable complete responses are rare with targeted therapies, most patients eventually have disease progression, and overall survival and toxicity profiles are suboptimal. Single-drug anti-PD-1 or PD-L1 immunotherapies proved efficacious in pretreated patients with advanced renal cell carcinoma, and subsequently investigative immunotherapy regimens (including combinations of immunotherapy drugs and combinations of immunotherapy drugs and tyrosine kinase inhibitors) showed notable antitumour activity in first-line advanced renal cell carcinoma; however, unfavourable toxicity was reported with some regimens combining immunotherapy drugs and tyrosine kinase inhibitors. The immunotherapy combination of nivolumab (monoclonal antibody against PD-1) plus ipilimumab (anti-CTLA-4 antibody) showed manageable safety and notable antitumour activity in the phase 1 CheckMate 016 trial of patients with advanced renal cell carcinoma. Building upon these results, the phase 3 CheckMate 214 trial showed a significant overall survival and objective response benefit with nivolumab plus ipilimumab compared with sunitinib in the first-line treatment of intermediate-risk or poor-risk patients (classified according to the International Metastatic Renal Cell ipilimumab (an anti-CTLA-4 antibody) is a new standard-of-care option for the first-line treatment of intermediate-risk or poor-risk patients with advanced

Carcinoma Database Consortium) with advanced renal cell carcinoma with a clear cell component. To our knowledge, CheckMate 214 was the first study to investigate the efficacy and safety of first-line nivolumab plus ipilimumab and the first to show a statistically significant overall survival benefit over sunitinib. On the basis of these results, the combination of nivolumab plus ipilimumab was approved by the European Commission and the US Food and Drug Administration for the first-line treatment of patients with intermediate-risk and poor-risk advanced renal cell carcinoma.

\section{Added value of this study}

Long-term updates crucially inform the value of immunotherapy-based regimens. Therefore, we report updated and key additional efficacy and safety data with longer follow-up. The superior long-term overall survival, late progression-free survival benefit, proportion of patients achieving complete response, and durability of response observed in the current analysis are encouraging. CheckMate 214 was the first phase 3 trial to investigate the efficacy and safety of first-line nivolumab plus ipilimumab in patients with advanced renal cell carcinoma, and the results presented are the first to show the long-term benefits of nivolumab plus ipilimumab in this patient population.

\section{Implications of all the available evidence}

Extended follow-up of first-line combination treatment with nivolumab plus ipilimumab in patients with advanced renal cell carcinoma continues to show increased antitumour activity compared with sunitinib in the CheckMate 214 trial. No new safety signals emerged with nivolumab plus ipilimumab, and the adverse event profile continued to be manageable. These results show the long-term benefits of nivolumab plus ipilimumab in patients with previously untreated advanced renal cell carcinoma. The long-term overall survival benefits, high proportions of complete response and ongoing response, and paucity of chronic toxicity with nivolumab plus ipilimumab after extended follow-up compares favourably with available reports of other combination regimens in first-line advanced renal cell carcinoma. Our results also support future analyses exploring the role for nivolumab plus ipilimumab in treating novel patient populations and ongoing studies combining nivolumab plus ipilimumab with novel drugs to further improve outcomes for patients with advanced renal cell carcinoma.
Healthcare, Charlotte, NC, USA (A Amin MD); Department of Medical Oncology, IRCCS San Matteo University Hospital Foundation, Pavia, Italy (C Porta MD); Department of Medicine, Roswell Park Cance Institute, Buffalo, NY, USA (S George MD); Department of Oncology, Davidoff Cancer Center, Rabin Medical Center, Petah Tikva, Israel and Tel Aviv University, Tel Aviv, Israel (V Neiman MD); Department of Oncology, Ospedale San Donato, Azienda USL Toscana Sud-Est, IstitutoToscanoTumori, Arezzo, Italy (Prof S Bracarda MD); Department of Medicine, University of Washington and Fred Hutchinson Cancer Research Center, Seattle, WA, USA (S S Tykodi MD); Department of Medical Oncology, Hôpitaux Universitaires de Strasbourg, Strasbourg, France (Prof P Barthélémy MD); Oncology Department, Sheba Medical Center, Ramat Gan, Israel (R Leibowitz-Amit MD); Department of Hematology and Oncology, Fox Chase Cancer Center, Philadelphia, PA, USA (E R Plimack MD); Department of Medical Oncology, University Medical Center Groningen, University of Groningen, Groningen, Netherlands (S F Oosting MD); Department of Internal Medicine, Division of Hematology and Oncology, University of Michigan Comprehensive Cancer Center, Ann Arbor, MI, USA (Prof B Redman DO); Department of Oncology, Palacky University, and University Hospital Olomouc, Olomouc, Czech Republic (Prof B Melichar MD); Department of Genitourinary Oncology, Barts Cancer Institute, Cancer Research UK Experimental Cancer Medicine Centre, Queen Mary University of London, Royal Free National Health Service Trust, London, UK (ProfT Powles MD), Department of Cancer Services: Oncology, Mount Vernon Cancer Centre, Northwood, Middlesex, UK (P Nathan MD); Department of Medical Oncology, Service de Cancérologie Médicale Hôpital Européen Georges Pompidou, Paris, France 
(Prof S Oudard MD); Department of Oncology, Monash Health, Melbourne, VIC, Australia (D Pook MD); Department of Genitourinary Oncology, Dana-Farber Cancer Institute, Brigham and Women's Hospital, Boston, MA, USA (T K Choueiri MD); Harvard Medical School, Boston, MA, USA (T K Choueiri); Department of Oncology, Aarhus University Hospital, Aarhus, Denmark (F Donskov MD); Department of Urology, Jena University Hospital, Jena, Germany (Prof M-O Grimm MD); Department of Medical Oncology, Westmead Hospital and Macquarie University, Sydney, NSW, Australia (H Gurney MD); Department of Oncology, Tom Baker Cancer Center, University of Calgary, Calgary, AB, Canada (D Y C Heng MD); Department of Medicine, British Columbia Cancer Agency, Vancouver, BC, Canada

(C K Kollmannsberger MD); Division of Medical Oncology, Duke Cancer Institute, Durham,

NC, USA (M R Harrison MD); Department of Urology,

Niigata University, Niigata, Japan (ProfYTomita MD);

Department of Medical Oncology, Hospital Universitario Marques de Valdecilla, IDIVAL, Santander, Spain (I Duran MD); Interdisciplinary Genitourinary Oncology at the West-German Cancer Center, Clinic for Internal Medicine (Tumor Research) and (linic for Urology, University Hospital Essen, Essen, Germany (ProfV Grünwald MD); Bristol-Myers Squibb,

Princeton, NJ, USA (M B McHenry PhD, $S$ Mekan MD); and Department of Genitourinary Medical Oncology, University of Texas, MD Anderson Cancer Center, Houston, TX, USA (Prof N M Tannir MD)

Correspondence to: Prof Robert J Motzer, Department of Medicine, Memorial Sloan Kettering Cancer Center, New York, NY 10021, USA motzerr@mskcc.org

See Online for appendix renal cell carcinoma, based on longer overall survival and a higher proportion of patients achieving an objective response compared with sunitinib (a tyrosine kinase inhibitor) shown in the randomised, phase 3 CheckMate 214 trial. $^{1-5}$

At a prespecified interim analysis with a minimum follow-up of 17.5 months, CheckMate 214 met two of three coprimary endpoints, with nivolumab plus ipilimumab showing longer overall survival and a higher proportion of patients achieving an objective response per independent radiology review committee (IRRC) compared with sunitinib in the primary efficacy population of patients with International Metastatic Renal Cell Carcinoma Database Consortium (IMDC) intermediate-risk and poor-risk advanced renal cell carcinoma. Median overall survival in this patient population was not reached (95\% CI 28.2 months-not estimable) with nivolumab and ipilimumab versus $26 \cdot 0$ months (95\% CI 22.1-not estimable) with sunitinib (hazard ratio [HR] 0.63 [99.8\% CI 0.44-0.89], $\mathrm{p}<0 \cdot 001$ ). Higher proportions of intermediate-risk and poor-risk patients achieved confirmed objective responses with nivolumab plus ipilimumab than with sunitinib (42\% vs $27 \%$, respectively; $\mathrm{p}<0.001$ ) and complete responses ( $9 \%$ vs $1 \%$; $\mathrm{p}<0 \cdot 001$ ). Median progression-free survival was 11.6 months $(95 \%$ CI $8 \cdot 7-15 \cdot 5)$ with the combination treatment versus 8.4 months $(7 \cdot 0-10 \cdot 8)$ with sunitinib-a clinically meaningful improvement that did not meet the $\alpha$ level $(\mathrm{p}<0.009)$ for statistical significance (HR for disease progression or death 0.82 [99.1\% CI $0.64-1 \cdot 05], p=0 \cdot 03)$. Among the secondary endpoints in the intention-to-treat population, including both intermediate-risk or poor-risk patients and favourable-risk patients, nivolumab plus ipilimumab showed longer overall survival (HR 0.68 [99.8\% CI $0 \cdot 49-0.95] ; \mathrm{p}<0.001)$, numerically higher objective res ponses (39\% vs 32\%; $\mathrm{p}=0.02$ ), and similar progressionfree survival (HR 0.98 [99.1\% CI 0.79-1.23]; $\mathrm{p}=0.85$ ) compared with sunitinib. In the exploratory favourablerisk subpopulation, the proportion of patients achieving an objective response was higher with sunitinib than with nivolumab and ipilimumab (29\% vs $52 \%$; $p<0 \cdot 001)$ and progression-free survival was longer (HR 2.18 [99.1\% CI 1.29-3.68]; $\mathrm{p}<0 \cdot 001$ ); however, for the overall survival analysis, only 37 deaths had occurred as of this database lock. ${ }^{1}$ Health-related quality of life was consistently better with nivolumab plus ipilimumab than with sunitinib in both the intention-to-treat population and in intermediate-risk or poor-risk patients. ${ }^{6}$

Long-term updates are crucial to inform the value of immunotherapy-based regimens. Targeted drugs have shown improved short-term endpoints, such as objective response and progression-free survival, ${ }^{7}$ but immunotherapy combinations are better assessed with endpoints indicative of durable response and long-term survival. In this Article, we report expanded CheckMate 214 efficacy and safety analyses with extended follow-up.

\section{Methods}

\section{Study design and participants}

CheckMate 214 was a randomised, open-label, phase 3 trial comparing nivolumab plus ipilimumab induction followed by nivolumab maintenance with sunitinib monotherapy in patients with previously untreated advanced renal cell carcinoma, recruited from 175 hospitals and cancer centres in 28 countries (appendix pp 2-5). Detailed methods have been reported previously. Eligible patients were aged 18 years or older, with histological confirmation of advanced or metastatic renal cell carcinoma with a clear cell component, and without previous systemic therapy for their disease, with the exception of one previous adjuvant or neoadjuvant therapy (not including VEGF-targeted drugs) for completely resectable renal cell carcinoma if recurrence occurred at least 6 months after the last dose. Further inclusion criteria were a Karnofsky performance status of at least $70 \%$ and measurable disease per Response Evaluation Criteria in Solid Tumors (RECIST) version 1.1. Key exclusion criteria were history of or current central nervous system metastases and active or history of autoimmune disease or use of systemic corticosteroids within 14 days of group assignment. Additionally, patients with any of the following laboratory test findings were deemed ineligible: white blood cell count of less than 2000 cells per $\mathrm{mm}^{3}$, neutrophil count of less than 1500 cells per $\mathrm{mm}^{3}$, platelets less than 100000 per $\mathrm{mm}^{3}$, aspartate aminotransferase or alanine aminotransferase more than 3-times the upper limit of normal (ULN; or $>5$-times ULN if liver metastases were present), total bilirubin more than 1.5-times ULN (except patients with Gilbert syndrome, who could have total bilirubin $<3.0 \mathrm{mg} / \mathrm{dL}$ ), serum creatinine more than 1.5 -times ULN, or creatinine clearance less than $40 \mathrm{~mL} / \mathrm{min}$.

CheckMate 214 was approved by the institutional review board or ethics committee at each site and conducted in accordance with Good Clinical Practice guidelines per the International Conference on Harmonisation. All patients provided written informed consent per Declaration of Helsinki principles. The protocol is available in the appendix (pp 15-146). Protocol deviations considered relevant were collected by treatment group and overall in all randomised patients (data not shown).

\section{Randomisation and masking}

Patients were randomly assigned (1:1) to nivolumab plus ipilimumab or sunitinib through an interactive voice response system. Randomisation was done via permuted blocks within each stratum, with a block size of four and stratified by IMDC risk score (favourable [0] vs intermediate [1-2] vs poor [3-6]) and by geographical region (USA vs Canada and Europe $v s$ rest of the world). ${ }^{1,8}$ Allocation and implementation was managed via the interactive voice response system. Patients and investigators were not masked to treatment assignment because this trial was open-label. 


\section{Procedures}

The treatment regimen was administered in cycles of 6 weeks. Nivolumab (3 mg/kg) and ipilimumab (1 mg/kg) were administered intravenously over approximately $60 \mathrm{~min}$ and $30 \mathrm{~min}$, respectively, every 3 weeks for four doses (induction), followed by nivolumab (3 mg/kg) every 2 weeks (maintenance). Sunitinib (50 mg) was administered orally once daily for 4 weeks on and 2 weeks off in each 6-week cycle. Treatment continued until disease progression or unacceptable toxicity. No dose reductions were allowed in the nivolumab plus ipilimumab group, whereas a maximum of two dose reductions in total were permitted for sunitinib in $12.5 \mathrm{mg}$ increments per day (such that the daily dose is not less than $25 \mathrm{mg}$ ) based on individual safety and tolerability. ${ }^{9}$ The data monitoring committee recommendation on Sept 6, 2016, was to stop the trial for superiority of overall survival with nivolumab plus ipilimumab versus sunitinib based on the predefined threshold for statistical significance. As a result, a protocol amendment on Nov 13, 2017, allowed for the following modifications based on recommendations of the study's independent data monitoring committee, which determined that the preplanned interim analysis results should be considered the final primary analysis results based upon the interim analysis of overall survival (appendix p 17): patients in the nivolumab plus ipilimumab group could discontinue after 2 years of study treatment even in the absence of disease progression or unacceptable toxicity at the discretion of the patient, investigator, or both; patients in the nivolumab plus ipilimumab group who were receiving nivolumab monotherapy could also switch to a flat dose of nivolumab (240 mg) every 2 weeks; and among intermediate-risk or poor-risk patients, crossover from the sunitinib group to the nivolumab plus ipilimumab group was allowed. Disease assessments were done with CT or MRI at baseline, and treated patients were evaluated for response according to RECIST version 1.1 guidelines beginning 12 weeks (or within 1 week either side of this timepoint) after randomisation and continuing every 6 weeks (or within 1 week either side of this timepoint) for the first 13 months, then every 12 weeks (or within 1 week either side of this timepoint) until disease progression or treatment discontinuation, whichever occurred later. Adverse events were assessed continuously during treatment visits, then at follow-up visits 1 and 2 and during overall survival follow-up, graded per National Cancer Institute Common Terminology Criteria for Adverse Events (CTCAE) version 4.0, ${ }^{10}$ and reported from the first dose, and up to and including 30 days after the last study treatment dose. Blood chemistry assessments were done on day 1 of weeks 1 and 4 during cycles 1 and 2; day 1 of weeks 1,3 , and 5 during subsequent cycles; and at follow-up visits 1 and 2 as detailed in the appendix ( $p$ 89).

\section{Outcomes}

The co-primary endpoints of CheckMate 214 were overall survival, progression-free survival per IRRC, and objective responses per IRRC in intermediate-risk and poor-risk patients. Secondary endpoints were overall survival, progression-free survival per IRRC, objective responses per IRRC in the intention-to-treat population (ie, all randomly assigned patients), and the incidence of adverse events in all treated patients. Exploratory endpoints included objective response, progression-free survival, and overall survival among favourable-risk patients, and safety in all treated patients (all reported in this Article), outcomes according to the level of tumour PD-L1, pharmacokinetics or pharmacodynamics of nivolumab, and health-related quality of life (not reported). Duration of treatment was reported for all treated patients. In the present prespecified follow-up analysis after the co-primary endpoints were met, overall survival was analysed as reported previously; ${ }^{1}$ however, progression-free survival and objective responses (including time to response and duration of response) were assessed per investigator using RECIST version 1.1 instead of IRRC in all risk subgroups and ITT patients. Overall survival was defined as the time from randomisation to the date of death. Objective response was defined as the proportion of enrolled and randomly assigned patients who achieved a best response of complete response or partial response according to RECIST version 1.1 based on investigator assessment. The primary definition of progression-free survival was specified as the time between the date of randomisation and the first date of documented progression, based on investigator assessments (per RECIST version 1.1), or death due to any cause, whichever occurred first. Patients who died without reported progression were considered to have progressed on the date of death. Prespecified safety outcomes included treatment-related adverse events and treatment-related adverse events leading to discontinuation or death, per CTCAE version $4.0 .^{10}$ Incidence of these adverse events was defined as the proportion of patients with any-grade adverse events in each treatment group. Treatment-related select adverse events (ie, prespecified and defined as events that might be immune-mediated, differ from those caused by non-immunotherapeutic drugs, might require immunosuppression for management, and whose early recognition might mitigate severe toxicity) were also reported, including skin, gastrointestinal, endocrine, hepatic, pulmonary, or renal system events. Additional details on the methods used for outcomes are reported in the appendix (p 6).

\section{Statistical analysis}

Details of the statistical analyses for the primary and secondary endpoints have been previously reported; since the first planned interim overall survival analysis met the prespecified statistical significance boundary for the co-primary endpoint of overall survival, it was considered the final primary analysis per protocol. ${ }^{1}$ Briefly, 1070 participants were estimated to be required to 
enrol 820 patients (ie, the number needed for robust statistical analysis) who were at intermediate or poor risk. The overall $\alpha$ of 0.05 was split among the co-primary endpoints $(0.001$ for the proportion of patients who achieved an objective response, 0.009 with $80 \%$ power for progression-free survival, and 0.04 with $90 \%$ power for overall survival) in the primary analysis. A formal comparison of overall survival in the intention-to-treat population was done using a two-sided stratified log-rank test at the same $\alpha$ at the time of the final analysis of co-primary endpoints based on a hierarchical testing procedure. Allocations $\alpha$ inform the sample size determination to adequately power the study and are no longer relevant in this analysis. We include $p$ values for descriptive purposes to confirm consistency with the primary analysis as appropriate. Efficacy outcomes were assessed in all randomised patients (the intention-totreat population and by disease risk level) and safety outcomes were assessed in all treated patients. The

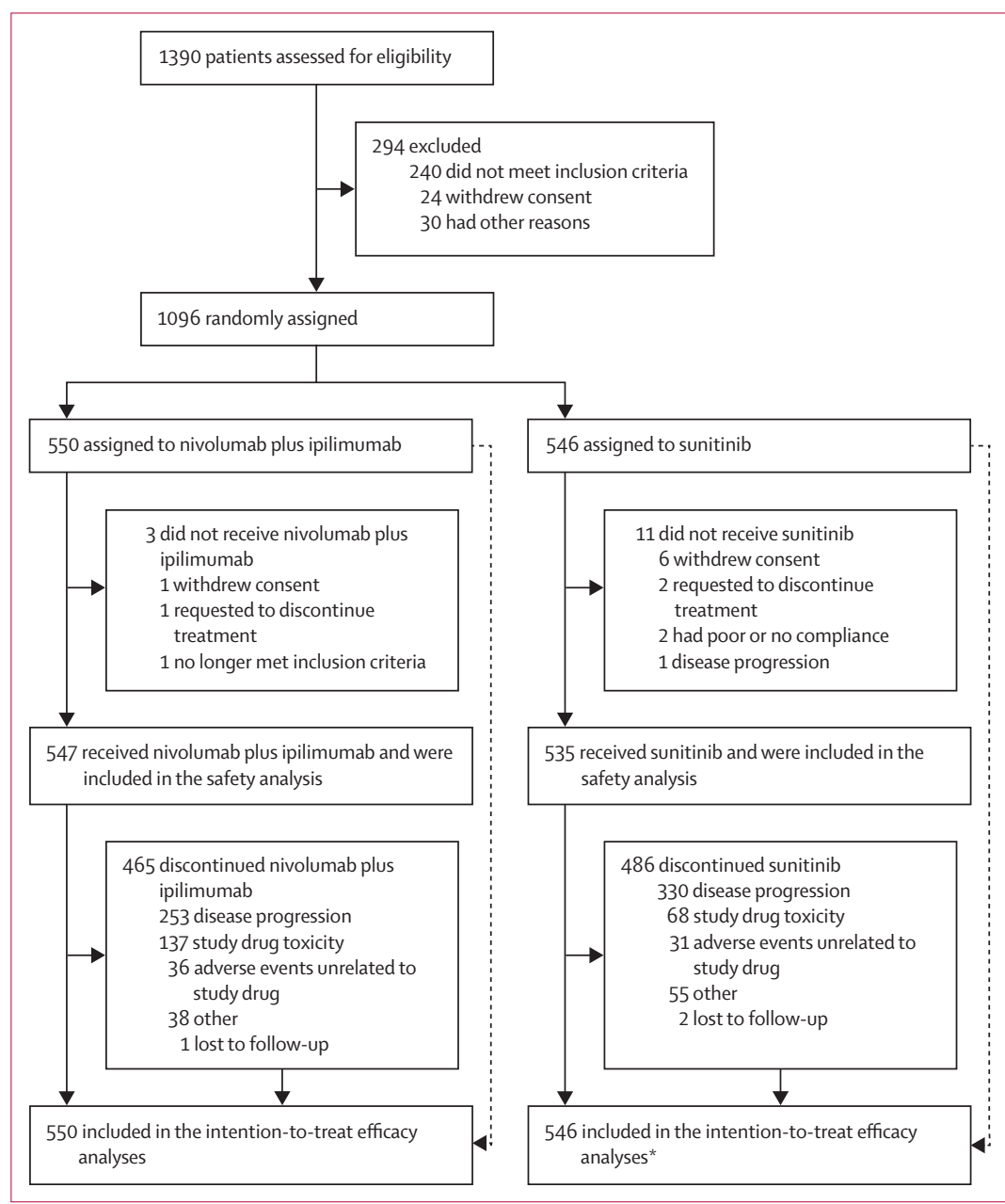

Figure 1: Trial profile

*11 intermediate-risk or poor-risk patients in the sunitinib group crossed over to the nivolumab plus ipilimumab group after the primary endpoint was assessed, but were not analysed as part of the nivolumab plus ipilimumab efficacy or safety population. prespecified analyses of overall survival, progression-free survival, duration of study therapy, and duration of response were estimated by use of the Kaplan-Meier method. ${ }^{11}$ For patients who were alive, their survival time was censored at the date of last contact; overall survival was censored for patients at the date of randomisation if they were randomly assigned to a treatment group but had no follow-up. Stratified Cox proportional HRs and 95\% CIs were calculated between treatment groups for overall survival and progression-free survival, and as an exploratory measure of duration of response. Post-hoc analysis of the effects of clinically relevant baseline features (tumour PD-L1 expression, neutrophil-tolymphocyte ratio, tumour burden, previous nephrectomy, individual IMDC risk factors, ${ }^{8}$ and individual Memorial Sloan Kettering Cancer Center risk factors ${ }^{12}$ ) on overall survival was done using univariable and multivariable models for each intention-to-treat treatment group separately to distinguish factors relevant to nivolumab plus ipilimumab versus sunitinib. Individual factors first underwent univariable analysis to preclude introducing collinearity into the model. A biologically plausible multivariable model was built and verified, as baseline factors associated with overall survival $(\mathrm{p}<0 \cdot 1)$ in the univariable model were entered into a full Cox proportional hazards multivariable regression model. Parsimonious (reduced) multivariable overall survival models included all baseline factors associated with overall survival at a nominal $\mathrm{p}$ value of less than $0 \cdot 05$. The proportion of patients achieving investigatorassessed objective response and the exact two-sided 95\% CIs were computed using the Clopper-Pearson method, ${ }^{13}$ with strata-adjusted (IMDC prognostic risk score $[0,1-2,3-6]$ and region [USA, Canada or Europe, and rest of the world]) objective response differences between treatment groups, based on DerSimonian-Laird methods, ${ }^{14}$ included as an exploratory measure. Additional post-hoc efficacy analyses in responders included baseline characteristics, summary of end of treatment patient status, depth of response, additional response kinetics, and treatment-free survival (defined as the time between protocol therapy discontinuation and subsequent systemic anticancer treatment initiation or death, whichever occurred first, in patients who discontinued protocol therapy for any reason). ${ }^{9}$ The prevalence of grade 3-4 treatment-related adverse events of clinical interest was analysed post hoc in all treated patients and graphed by system organ class using density plots summing vectors over time for each treatment group, in which each vector represents an individual patient's time to onset through resolution of the adverse event; if multiple events were reported for a patient in the same organ category, the earliest and the latest to resolve in the category were used.

All statistical analyses were done with SAS version 8.2 or East version 5.4. This study is registered with ClinicalTrials.gov, number NCT02231749. 


\section{Role of the funding source}

The funders contributed to the study design, data analysis, and data interpretation in collaboration with the authors. The funders had no role in data collection. The funders provided financial support for editorial and writing assistance. All authors had full access to all of the data included in the study. The corresponding author had full access to all of the data and had final responsibility for the decision to submit for publication.

\section{Results}

Between Oct 16, 2014, and Feb 23, 2016, 1390 patients were screened for enrolment into the CheckMate 214 trial; of the 1390 patients assessed, 240 (17\%) did not meet the inclusion criteria, 24 (2\%) withdrew consent, and $30(2 \%)$ were not randomly assigned because of other reasons (figure 1). 1096 (79\%) patients (intention-to-treat population) were randomly assigned to nivolumab plus ipilimumab (550 patients; 425 at intermediate or poor risk and 125 at favourable risk) or sunitinib (546 patients; 422 at intermediate or poor risk, 124 at favourable risk). 547 patients in the nivolumab plus ipilimumab group and 535 in the sunitinib group received the assigned treatment (ie, per-protocol population) and were included in the safety analysis. Baseline characteristics were similar in the two treatment groups, and across intermediate-risk or poor-risk patients, the intention-to-treat population, and favourable-risk patients (appendix p 7). However, baseline tumour PD-L1 expression was lower and the incidence of previous nephrectomy was higher in both treatment groups in favourable-risk patients than in the intermediate-risk or poor-risk group and the intention-totreat population. ${ }^{1}$ The data cutoff for this analysis with extended follow-up was Aug 6, 2018. At a minimum overall survival follow-up of 30 months (median $32 \cdot 4$ months [IQR $13 \cdot 4-36 \cdot 3]), 82$ (15\%) of 547 patients continued therapy in the nivolumab plus ipilimumab group and 49 (9\%) of 535 patients in the sunitinib group (figure 1). As of the database lock, 11 intermediate-risk or poor-risk patients crossed over from sunitinib to nivolumab plus ipilimumab, per a protocol amendment that provided this option for second-line nivolumab plus ipilimumab treatment as a part of the study.

Median duration of treatment was 7.9 months $(95 \%$ CI 6.5-8.4) in the nivolumab plus ipilimumab group and 7.8 months $(6 \cdot 4-8 \cdot 6)$ in the sunitinib group. Treated patients in the nivolumab plus ipilimumab group received a median of 14.0 doses (IQR $4 \cdot 0-43 \cdot 0$ ) of nivolumab and $4.0(4 \cdot 0-4 \cdot 0)$ doses of ipilimumab. The median average daily dose of sunitinib received was 46.4 mg (IQR 37.0-53.6) over the 28-day cycle. Dose reductions occurred in 286 (53\%) of the 535 patients treated with sunitinib, primarily because of adverse events (278 patients).

In the primary efficacy population of intermediate-risk or poor-risk patients, $182(43 \%)$ of 425 patients died in the nivolumab plus ipilimumab group versus

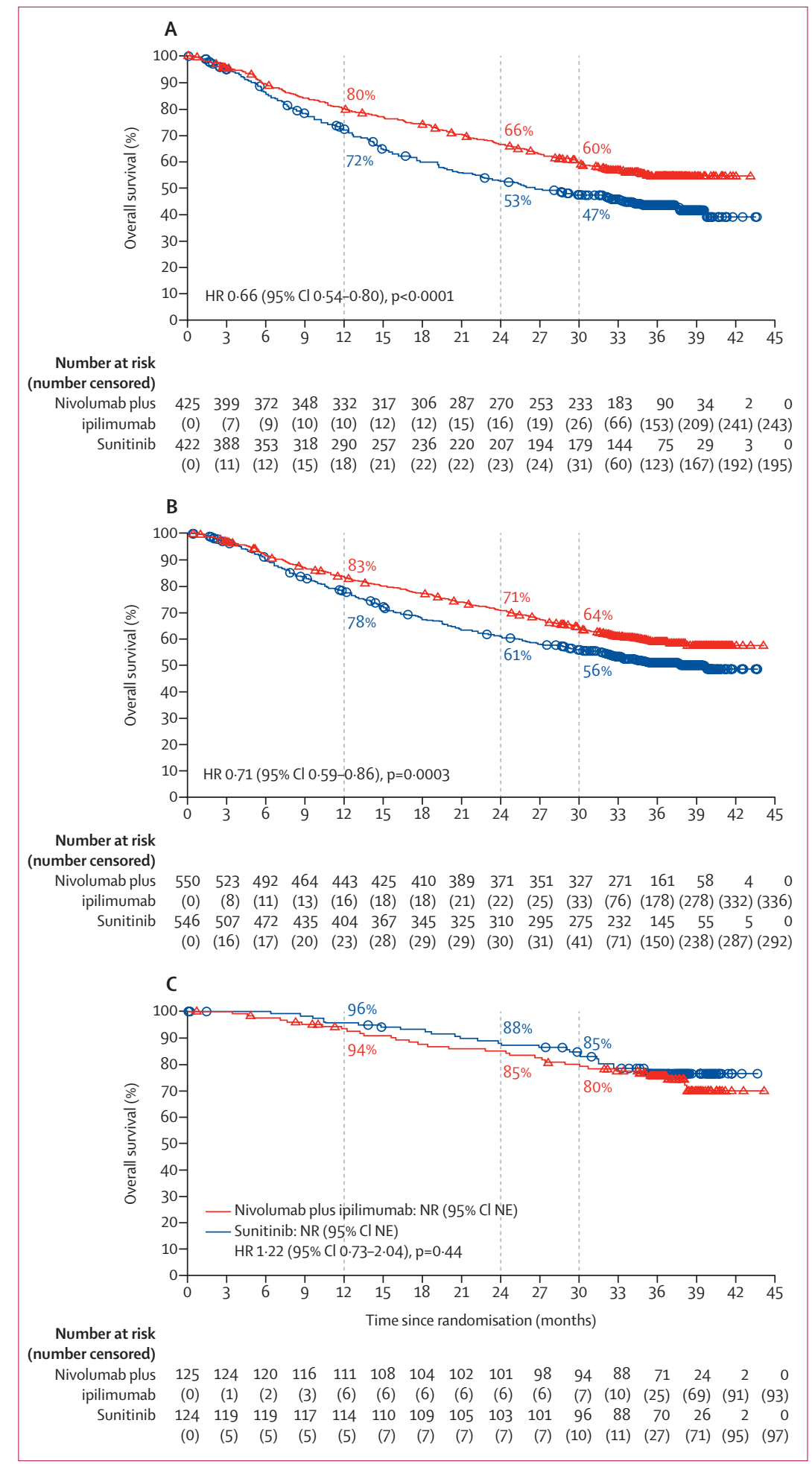

Figure 2: Overall survival in IMDC intermediate-risk or poor-risk patients $(A)$, in the intention-to-treat population (B), and in IMDC favourable-risk patients (C)

$\mathrm{HR}=$ hazard ratio. IMDC=International Metastatic Renal Cell Carcinoma Database Consortium. NE=not evaluable. $\mathrm{NR}=$ not reached. Red triangles and blue circles represent censored patients. 


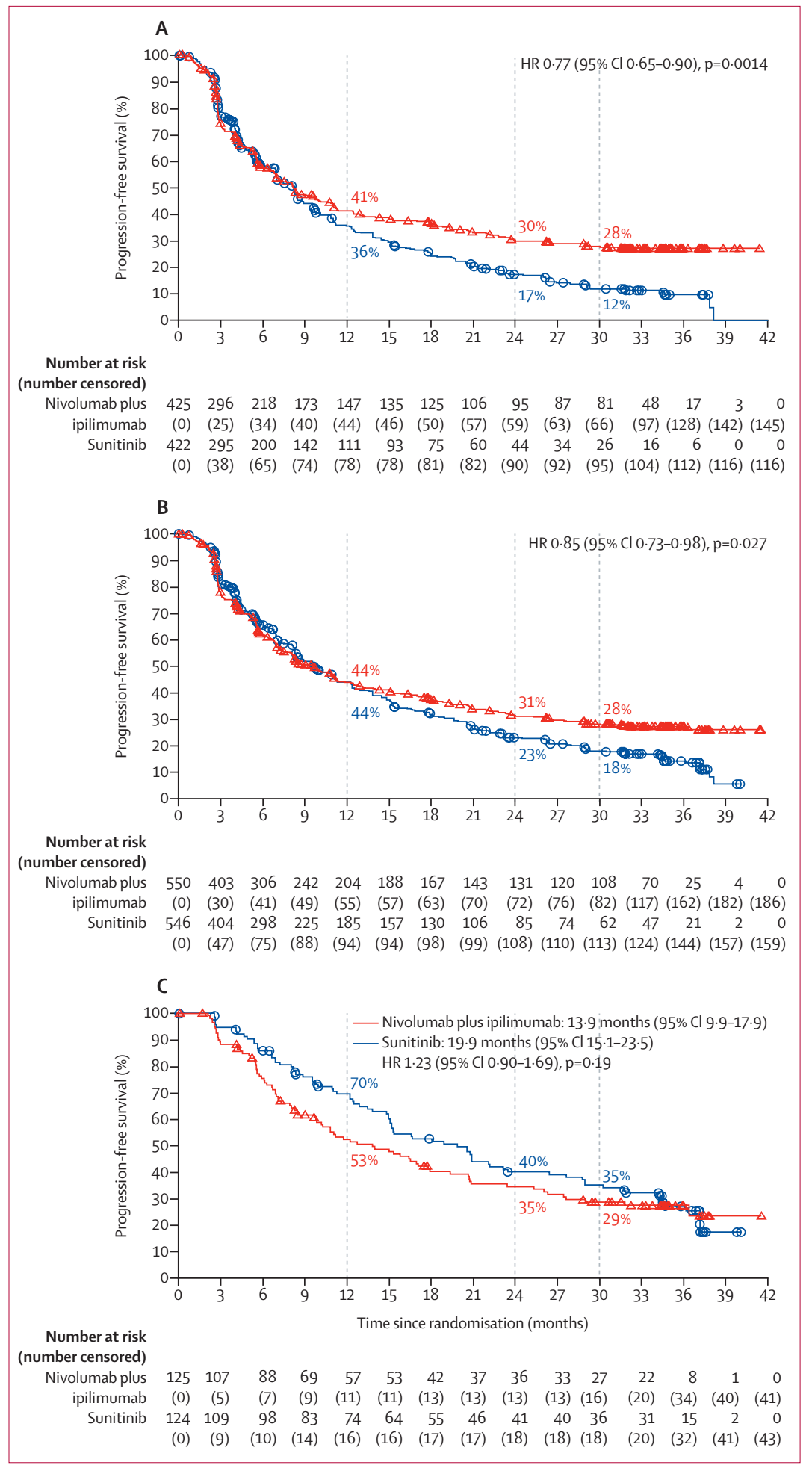

Figure 3: Progression-free survival per investigator assessment in IMDC intermediate-risk or poor-risk patients (A), in the intention-to-treat population (B), and in IMDC favourable-risk patients (C) $\mathrm{HR}=$ hazard ratio. IMDC=International Metastatic Renal Cell Carcinoma Database Consortium. Red triangles and blue circles represent censored patients.
227 (54\%) of 422 in the sunitinib group. Median overall survival was not reached (95\% CI 35.6-not estimable) with nivolumab plus ipilimumab versus $26 \cdot 6$ months $(22 \cdot 1-33 \cdot 4)$ with sunitinib (hazard ratio [HR] 0.66 [95\% CI $0 \cdot 54-0 \cdot 80], \mathrm{p}<0 \cdot 0001$ ) and 30-month overall survival was $60 \%$ (95\% CI 55-64) versus $47 \%$ (43-52), respectively (figure 2A). In the intention-to-treat (secondary efficacy) population, 214 (39\%) of 550 patients died in the nivolumab plus ipilimumab group versus $254(47 \%)$ of 546 in the sunitinib group. Median overall survival was not reached (95\% CI not estimable) with nivolumab and ipilimumab versus 37.9 months (32.2-not estimable) with sunitinib (HR $0 \cdot 71$ [95\% CI $0 \cdot 59-0 \cdot 86], \mathrm{p}=0 \cdot 0003)$ and 30 -month overall survival was $64 \%(60-68)$ versus $56 \%(52-60)$, respectively (figure 2B). In favourable-risk patients (the exploratory efficacy population), 32 (26\%) of 125 patients in the nivolumab plus ipilimumab group versus 27 (22\%) of 124 in the sunitinib group died. Overall survival was similar in the two groups, with 30-month overall survival of $80 \%$ (72-86) with nivolumab plus ipilimumab versus $85 \%$ (77-90) with sunitinib (figure 2C).

In intermediate-risk or poor-risk patients, 275 (65\%) of 425 patients in the nivolumab plus ipilimumab group versus 304 (72\%) of 422 patients in the sunitinib group had a progression event. Median progression-free survival was 8.2 months $(95 \%$ CI $6 \cdot 9-10 \cdot 0)$ with nivolumab plus ipilimumab versus 8.3 months $(7 \cdot 0-8 \cdot 8)$ with sunitinib (HR 0.77 [95\% CI 0.65-0.90], $\mathrm{p}=0 \cdot 0014$ ). The progressionfree survival curves for the two treatment groups only began to separate after around 9 months (figure 3A), and the 30 -month progression-free survival probability was $28 \%$ (95\% CI 23-33) in the nivolumab plus ipilimumab group versus $12 \%(8-16)$ in the sunitinib group. In the intention-to-treat population (secondary efficacy population), 357 (65\%) of 550 who received nivolumab plus ipilimumab versus 385 (71\%) of 546 who received sunitinib had a progression event. Median progressionfree survival was 9.7 months $(95 \%$ CI $8 \cdot 1-11 \cdot 1)$ with nivolumab plus ipilimumab versus 9.7 months $(8 \cdot 3-11 \cdot 1)$ with sunitinib (HR 0.85 [95\% CI 0.73-0.98], $\mathrm{p}=0 \cdot 027$ ). Differences in investigator-assessed progression-free survival between the two groups only began to emerge after 12 months (figure 3B). A plateau emerged at 30 months with nivolumab plus ipilimumab treatment. 30 -month progression-free survival probability was $28 \%$ (95\% CI 24-32) with nivolumab plus ipilimumab versus $18 \%$ (14-22) with sunitinib (figure 3B).

In favourable-risk patients, $82(66 \%)$ of 125 patients in the nivolumab plus ipilimumab group had a progression event versus 81 (65\%) of 124 in the sunitinib group. Progression-free survival did not differ significantly between treatment groups: 30-month progression-free survival was $29 \%$ (21-38) with nivolumab plus ipilimumab versus 35\% (26-44) with sunitinib (figure 3C).

In the intermediate-risk or poor-risk patients, the proportion of patients achieving an investigator-assessed 


\begin{tabular}{|c|c|c|c|c|c|c|c|c|c|}
\hline & \multicolumn{3}{|c|}{ IMDC intermediate-risk or poor-risk patients } & \multicolumn{3}{|c|}{ Intention-to-treat population } & \multicolumn{3}{|c|}{ IMDC favourable-risk patients } \\
\hline & $\begin{array}{l}\text { Nivolumab plus } \\
\text { ipilimumab }(n=425)\end{array}$ & $\begin{array}{l}\text { Sunitinib } \\
(n=422)\end{array}$ & $\mathrm{p}$ value & $\begin{array}{l}\text { Nivolumab plus } \\
\text { ipilimumab }(n=550)\end{array}$ & $\begin{array}{l}\text { Sunitinib } \\
(\mathrm{n}=546)\end{array}$ & $\mathrm{p}$ value & $\begin{array}{l}\text { Nivolumab plus } \\
\text { ipilimumab ( } n=125)\end{array}$ & $\begin{array}{l}\text { Sunitinib } \\
(\mathrm{n}=124)\end{array}$ & p value \\
\hline $\begin{array}{l}\text { Proportion of patients with } \\
\text { confirmed objective responses, \% } \\
(95 \% \mathrm{Cl})\end{array}$ & $42 \%(37-47)$ & $29 \%(25-34)$ & 0.0001 & $41 \%(37-46)$ & $34 \%(30-38)$ & 0.015 & $39 \%(31-48)$ & $50 \%(41-59)$ & 0.14 \\
\hline \multicolumn{10}{|l|}{ Best overall response } \\
\hline Complete response & $48(11 \%)$ & $5(1 \%)$ & .. & $58(11 \%)$ & $10(2 \%)$ & .. & $10(8 \%)$ & $5(4 \%)$ &.$\cdot$ \\
\hline Partial response & $130(31 \%)$ & $119(28 \%)$ & & $169(31 \%)$ & $176(32 \%)$ & .. & $39(31 \%)$ & $57(46 \%)$ & \\
\hline Stable disease & $110(26 \%)$ & $174(41 \%)$ &.. & $165(30 \%)$ & $222(41 \%)$ & .. & $55(44 \%)$ & $48(39 \%)$ &. \\
\hline Progressive disease & $106(25 \%)$ & $80(19 \%)$ &.. & $121(22 \%)$ & $86(16 \%)$ &. & $15(12 \%)$ & $6(5 \%)$ &. \\
\hline Unable to determine or not reported & $31(7 \%)$ & $44(10 \%)$ & .. & $37(7 \%)$ & $52(10 \%)$ &. & $6(5 \%)$ & $8(6 \%)$ &. \\
\hline $\begin{array}{l}\text { Median time to confirmed objective } \\
\text { response (IQR), months* }\end{array}$ & $\begin{array}{l}\mathrm{n}=176 \\
2 \cdot 8(2 \cdot 7-3 \cdot 1)\end{array}$ & $\begin{array}{l}\mathrm{n}=124 \\
4 \cdot 0(2 \cdot 8-5 \cdot 5)\end{array}$ &. & $\begin{array}{l}\mathrm{n}=225 \\
2 \cdot 8(2 \cdot 7-4 \cdot 0)\end{array}$ & $\begin{array}{l}n=186 \\
4 \cdot 0(2 \cdot 8-6 \cdot 0)\end{array}$ & .. & $\begin{array}{l}\mathrm{n}=49 \\
2 \cdot 9(2 \cdot 7-5 \cdot 5)\end{array}$ & $\begin{array}{l}\mathrm{n}=62 ; \\
4 \cdot 2(2 \cdot 8-6 \cdot 9)\end{array}$ & .. \\
\hline $\begin{array}{l}\text { Median time to confirmed complete } \\
\text { response (IQR), months }\end{array}$ & $\begin{array}{l}\mathrm{n}=48 \\
5 \cdot 8(2 \cdot 9-10 \cdot 5)\end{array}$ & Not calculated &. & $\begin{array}{l}\mathrm{n}=58 \\
7 \cdot 6(3 \cdot 8-11 \cdot 2)\end{array}$ & Not calculated & .. & $\begin{array}{l}\mathrm{n}=10 \\
10 \cdot 5(7 \cdot 0-14 \cdot 0)\end{array}$ & Not calculated &.. \\
\hline $\begin{array}{l}\text { Patients with duration of response } \\
\geq 18 \text { months* }\end{array}$ & $92 / 176(52 \%)$ & $35 / 124(28 \%)$ & .. & $120 / 225(53 \%)$ & $72 / 186(39 \%)$ & .. & $28 / 49(57 \%)$ & $37 / 62(60 \%)$ & .. \\
\hline Patients with ongoing response* & $104 / 176(59 \%)$ & $43 / 124(35 \%)$ &. & $131 / 225(58 \%)$ & $69 / 186(37 \%)$ & .. & $27 / 49(55 \%)$ & $26 / 62(42 \%)$ &.. \\
\hline $\begin{array}{l}\text { Patients with ongoing complete } \\
\text { response }\end{array}$ & $42 / 48(88 \%)$ & $4 / 5(80 \%)$ &. & $51 / 58(88 \%)$ & $6 / 10(60 \%)$ & .. & $9 / 10(90 \%)$ & $2 / 5(40 \%)$ &. \\
\hline
\end{tabular}

confirmed objective response was higher in the nivolumab plus ipilimumab group than in the sunitinib group (178 [42\%] of 425 vs 124 [29\%] of 422; $\mathrm{p}=0 \cdot 0001$ ). Similarly, in the intention-to-treat population, the proportion of patients achieving an investigator-assessed confirmed objective response was higher in the nivolumab plus ipilimumab group than in the sunitinib group (227 [41\%] of 550 vs 186 [34\%] of 546, p=0 - 015). The proportion of patients achieving an investigator-assessed confirmed objective response with nivolumab plus ipilimumab was consistent across risk groups, whereas the proportion achieving an objective response with sunitinib was lowest among patients with worse IMDC prognosis (table 1). Depth of response was greater with nivolumab plus ipilimumab versus sunitinib, as measured in the proportion of patients in the intentionto-treat population who achieved a $50 \%$ or greater best tumour burden reduction (185 [34\%] of 550 vs 114 [21\%] of 546). Additionally, the proportion of patients achieving a complete response was consistently higher with nivolumab plus ipilimumab than with sunitinib across all risk categories (table 1). The proportion of patients achieving a partial response was similar between groups, with the exception of favourable-risk patients in the sunitinib group (table 1). Strata-adjusted differences between the nivolumab plus ipilimumab and sunitinib treatment groups in the proportion of patients achieving an objective response were $12 \cdot 8 \%(95 \%$ CI $6 \cdot 5-19 \cdot 2)$ in intermediate-risk or poor-risk patients, $7 \cdot 6 \%(2 \cdot 0-13 \cdot 2)$ in the intention-to-treat population, and $-11.0 \%$ $(-12 \cdot 0$ to $1 \cdot 0)$ in favourable-risk patients.
A multivariable model was used to assess the effect of baseline clinical features on overall survival. Baseline factors were first analysed individually in the univariable analysis to preclude introducing collinearity into the model (appendix pp 8, 9). In the reduced multivariable models we observed a similar significant negative prognostic effect of the following baseline clinical risk factors on overall survival in the intention-to-treat population in both the nivolumab plus ipilimumab and sunitinib groups (table 2): lower Karnofsky performance status, higher lactate dehydrogenase concentration, higher neutrophil:lymphocyte ratio, and higher sum of reference diameters of target lesions. Higher corrected calcium concentration only significantly affected overall survival in the nivolumab plus ipilimumab group, whereas lower haemoglobin concentration, higher tumour PD-L1 expression, and no previous nephrectomy only significantly affected overall survival in the sunitinib group (table 2).

In the intention-to-treat patients (secondary efficacy population) in the nivolumab plus ipilimumab group, baseline characteristics for responders $(n=227)$, complete responders $(n=58)$, and non-responders $(n=323)$ were mostly similar (ad-hoc analysis; appendix p 10). However, responders and complete responders to nivolumab plus ipilimumab generally had lower disease burdens, including fewer sites with lesions and less bone and kidney involvement versus non-responders. Additionally, partial responders and complete responders to nivolumab plus ipilimumab both had higher baseline tumour PD-L1 expression than non-responders (appendix p 10). Data 


\begin{tabular}{|c|c|c|c|c|c|c|}
\hline & $\mathrm{n}$ & $\begin{array}{l}\text { Median overall } \\
\text { survival, months } \\
(95 \% \mathrm{Cl})\end{array}$ & $\begin{array}{l}\text { 24-month overall } \\
\text { survival, \% }(95 \% \mathrm{Cl})\end{array}$ & $\begin{array}{l}\text { 30-month overall } \\
\text { survival, } \%(95 \% \mathrm{Cl})\end{array}$ & $\mathrm{HR}(95 \% \mathrm{Cl})$ & p value \\
\hline \multicolumn{7}{|c|}{ Nivolumab plus ipilimumab group $(n=550)$} \\
\hline Karnofsky performance status, \% &.. & .. & .. & .. & $2.80(1.93-4.06)$ & $<0.0001$ \\
\hline$\leq 70 \%$ & 55 & $16 \cdot 8(8 \cdot 5-22 \cdot 0)$ & $34 \%(21-47)$ & $32 \%(20-45)$ & .. & .. \\
\hline$>70 \%$ & 495 & $\mathrm{NR}(\mathrm{NE})$ & $75 \%(70-78)$ & $68 \%(63-72)$ & .. & .. \\
\hline $\begin{array}{l}\text { Corrected calcium concentration, } \\
\mathrm{mg} / \mathrm{dL}\end{array}$ &.. & .. & .. &.. & $0.63(0.46-0.86)$ & 0.0042 \\
\hline$\leq 10$ & 455 & $\mathrm{NR}(\mathrm{NE})$ & $73 \%(68-77)$ & $68 \%(63-72)$ & .. & .. \\
\hline$>10$ & 95 & $29 \cdot 9(24 \cdot 3-\mathrm{NE})$ & $61 \%(50-70)$ & $49 \%(39-59)$ &.. & .. \\
\hline Lactate dehydrogenase concentration &.. & .. & .. & .. & $2 \cdot 02(1 \cdot 22-3 \cdot 34)$ & 0.0063 \\
\hline$>1 \cdot 5$-times ULN & 27 & $16 \cdot 7(5 \cdot 5-28 \cdot 9)$ & $40 \%(21-58)$ & $26 \%(10-45)$ &. &.$\cdot$ \\
\hline$\leq 1 \cdot 5$-times ULN & 515 & NR (NE) & $72 \%(68-76)$ & $66 \%(62-70)$ &.. & .. \\
\hline Neutrophil-to-lymphocyte ratio &.. & .. & .. & .. & $1.64(1 \cdot 24-2 \cdot 17)$ & 0.0006 \\
\hline$\geq 2 \cdot 9$ & 275 & NR (29·8-NE) & $62 \%(56-68)$ & $56 \%(50-61)$ & .. & .. \\
\hline$<2 \cdot 9$ & 274 & $\mathrm{NR}(\mathrm{NE})$ & $79 \%(74-84)$ & $73 \%(67-78)$ & .. & .. \\
\hline $\begin{array}{l}\text { Median sum of reference diameters } \\
\text { of target lesions, } \mathrm{mm}\end{array}$ &.. & .. & .. & .. & $0.55(0.41-0.75)$ & 0.0001 \\
\hline$<64 \cdot 0$ & 268 & $\mathrm{NR}(\mathrm{NE})$ & $81 \%(76-86)$ & $75 \%(70-80)$ & .. & .. \\
\hline$\geq 64 \cdot 0$ & 282 & $32 \cdot 8(28 \cdot 2-\mathrm{NE})$ & $61 \%(55-66)$ & $54 \%(48-60)$ & .. & .. \\
\hline \multicolumn{7}{|l|}{ Sunitinib group $(n=546)$} \\
\hline Karnofsky performance status, $\%$ &.. & .. & .. & .. & $1.98(1.35-2.92)$ & 0.0005 \\
\hline$\leq 70 \%$ & 50 & $12 \cdot 9(6 \cdot 1-26 \cdot 3)$ & $39 \%(26-53)$ & $33 \%(20-46)$ & .. & .. \\
\hline$>70 \%$ & 496 & NR (33.4-NE) & $63 \%(58-67)$ & $58 \%(54-63)$ & .. &.$\cdot$ \\
\hline Haemoglobin concentration &.. &.. & .. & .. & $1.78(1 \cdot 35-2 \cdot 34)$ & $<0.0001$ \\
\hline$<L L N$ & 249 & $20 \cdot 0(15 \cdot 7-27 \cdot 0)$ & $47 \%(41-53)$ & $41 \%(35-47)$ & .. & .. \\
\hline$\geq \mathrm{LLN}$ & 294 & NR (NE) & $73 \%(67-77)$ & $69 \%(63-74)$ & .. &.. \\
\hline Lactate dehydrogenase concentration &.. & .. & .. &.$\cdot$ & $4.02(2.45-6.59)$ & $<0.0001$ \\
\hline$>1 \cdot 5$-times ULN & 22 & $4 \cdot 4(2 \cdot 0-10 \cdot 3)$ & $11 \%(2-28)$ & $11 \%(2-28)$ & .. & .. \\
\hline$\leq 1 \cdot 5$-times ULN & 521 & NR (34·3-NE) & $63 \%(59-67)$ & $58 \%(54-62)$ &. &.$\cdot$ \\
\hline Neutrophil-to-lymphocyte ratio &.. & .. & .. & .. & $1.82(1 \cdot 38-2 \cdot 39)$ & $<0.0001$ \\
\hline$\geq 2 \cdot 9$ & 271 & $22 \cdot 4(17 \cdot 7-29 \cdot 0)$ & $49 \%(42-55)$ & $43 \%(37-49)$ & .. & .. \\
\hline$<2 \cdot 9$ & 271 & NR (NE) & $73 \%(67-78)$ & $69 \%(63-74)$ & .. &.$\cdot$ \\
\hline PD-L1 expression status, \% &.. & .. & .. & .. & $0.70(0.52-0.93)$ & 0.014 \\
\hline$<1 \%$ & 376 & NR (34·8-NE) & $65 \%(60-70)$ & $60 \%(55-65)$ & .. & .. \\
\hline$\geq 1 \%$ & 127 & $23 \cdot 9(15 \cdot 8-34 \cdot 4)$ & $49 \%(40-58)$ & $44 \%(35-53)$ &.$\cdot$ &.. \\
\hline Previous nephrectomy & .. &.$\cdot$ & .. & .. & $1.44(1.06-1.96)$ & 0.019 \\
\hline No & 109 & $15 \cdot 7(13 \cdot 0-25 \cdot 8)$ & $41 \%(32-51)$ & $36 \%(27-45)$ & .. & .. \\
\hline Yes & 437 & NR (39.7-NE) & $65 \%(61-70)$ & $61 \%(56-65)$ & .. &.. \\
\hline $\begin{array}{l}\text { Median sum of reference diameters } \\
\text { of target lesions, } \mathrm{mm}\end{array}$ &.. &.$\cdot$ &.$\cdot$ &.$\cdot$ & $0.69(0.52-0.91)$ & 0.0085 \\
\hline$<64.0$ & 275 & NR (39.7-NE) & $71 \%(65-76)$ & $67 \%(61-72)$ & .. & .. \\
\hline$\geq 64 \cdot 0$ & 269 & $24(19 \cdot 2-30 \cdot 1)$ & $50 \%(44-56)$ & $44 \%(38-50)$ & .. & .. \\
\hline
\end{tabular}

for sunitinib were not described in this Article, because they have been reported in this population elsewhere. ${ }^{15,16}$

Median time to confirmed objective response is shown in table 1 and was shorter in the nivolumab plus ipilimumab group than in the sunitinib group; 169 (75\%) of 225 responders to nivolumab plus ipilimumab in the intention-to-treat population achieved a response by 4 months (based on the upper limit of the IQR [75\%] for responders equaling 4 months; table 1). Among all complete responders to nivolumab plus ipilimumab in the intention-to-treat population, three $(5 \%)$ of 58 achieved a complete response at the first scan, whereas most converted from partial response (44 [76\%] of 58 at a median of 6.9 months [IQR 3.2-9.1]) or from stable disease (11 [19\%] of 58 at a median of $11 \cdot 3$ months [IQR 3.8-15 -4]). In the nivolumab plus ipilimumab group, there were 
six additional complete responses since the previous database lock in 2017, and 44 (75\%) of 58 complete responses were reached by $11 \cdot 2$ months (table 1 ).

Duration of response was longer with nivolumab plus ipilimumab than with sunitinib in the intention-to-treat population (figure 4). The Kaplan-Meier duration of response curve reached a plateau above $50 \%$ in the nivolumab plus ipilimumab group, whereas the probability of maintained response with sunitinib continued to decrease over time to the end of follow-up (figure 4). A higher proportion of all responders to nivolumab plus ipilimumab than to sunitinib achieved a durable response lasting at least 18 months and had ongoing response as of the database lock (table 1 ).

The median duration of treatment in responders was 20.6 months (95\% CI 17.7-23.2) in the nivolumab plus ipilimumab group versus 21.2 months $(18 \cdot 9-24 \cdot 4)$ in the sunitinib group. In responders who discontinued study therapy across all risk categories, higher proportions of patients in the nivolumab plus ipilimumab group did not continue onto subsequent therapy than in the sunitinib group, including among responders with ongoing treatment-free survival (68 [38\%] of 178 vs 32 [26\%] of 124 intermediate-risk or poor-risk patients and 18 [37\%] of 49 vs 12 [19\%] of 62 favourable-risk patients, respectively; appendix p 14).

In the intention-to-treat population, 264 (48\%) of 550 patients in the nivolumab plus ipilimumab group and 334 (61\%) of 546 in the sunitinib group received subsequent systemic therapy; most commonly sunitinib (120 [22\%]), pazopanib (95 [17\%]), axitinib (86 [16\%]), and cabozantinib (61 [11\%]) in the nivolumab plus ipilimumab group; and nivolumab (192 [35\%]), axitinib (117 [21\%]), sunitinib (65 [12\%]), and everolimus (60 [11\%]) in the sunitinib group.

No new safety signals emerged with longer follow-up. Similar proportions of patients had treatment-related adverse events of any grade in the nivolumab plus ipilimumab group compared with the sunitinib group (513 [94\%] of 547 patients vs 521 [97\%] of 535 patients; appendix p 11). Fewer grade 3 or 4 treatment-related adverse events occurred with nivolumab plus ipilimumab than with sunitinib (255 [47\%] of 547 vs 342 [64\%] of 535; appendix p 11). The most common grade 3-4 treatmentrelated adverse events in the nivolumab and ipilimumab group were increased lipase (57 [10\%] of 547), increased amylase (31[6\%]), and increased alanine aminotransferase (28 [5\%]), whereas in the sunitinib group they were hypertension (90 [17\%] of 535), fatigue (51 [10\%]), and palmar-plantar erythrodysaesthesia (49 [9\%]). Any-grade treatment-related adverse events occurring in more than $15 \%$ of patients in either group with treatment-related grade 3-4 adverse events are shown in figure 5A. Tracking the most common organ classes of high-grade treatmentrelated adverse events over time, differences in early and chronic toxicity burden between groups were observed, with sunitinib toxicity continuing despite dose

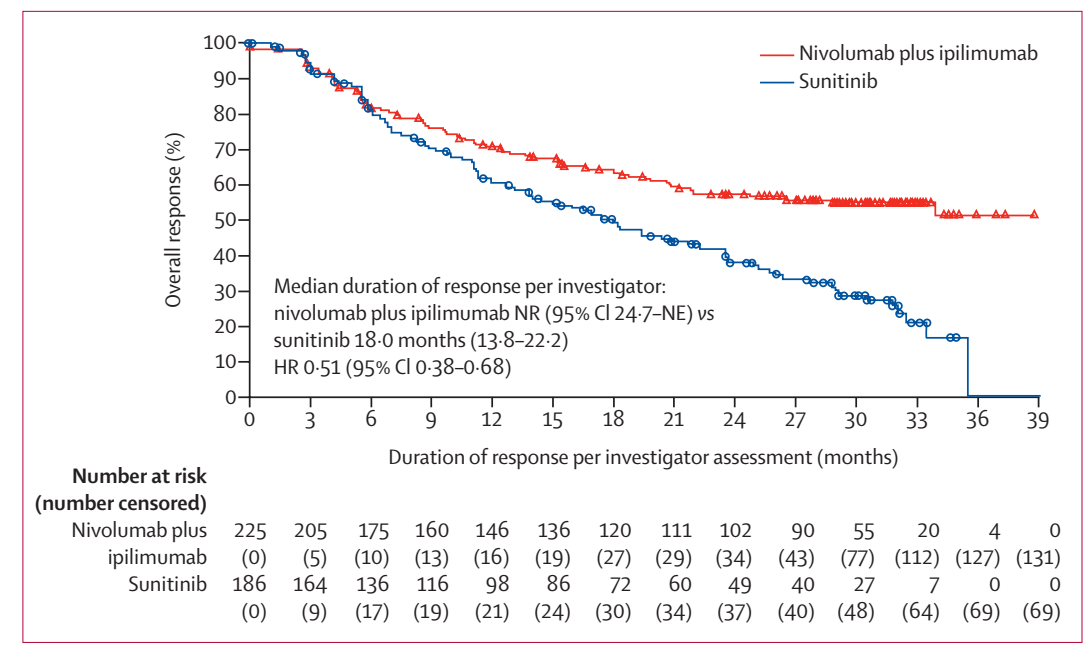

Figure 4: Duration of response in the nivolumab plus ipilimumab and sunitinib groups $\mathrm{HR}=$ hazard ratio. $\mathrm{NE}=$ not evaluable. $\mathrm{NR}=$ not reached.

adjustments, whereas the overall toxicity burden in the nivolumab plus ipilimumab group was low (figure $5 \mathrm{~B}, 5 \mathrm{C}$ ). More grade 3 or 4 treatment-related adverse events occurred during the combination induction versus the maintenance phase in the nivolumab plus ipilimumab group (figure 5B).

Treatment-related adverse events leading to discontinuation occurred in 119 (22\%) of 547 patients in the nivolumab plus ipilimumab group, most commonly due to increased alanine aminotransferase (15 [3\%]), diarrhoea (14 [3\%]), and increased aspartate aminotransferase (12 [2\%]); the majority of these patients discontinued after completing the nivolumab plus ipilimumab induction phase (data not shown). Treatment-related adverse events leading to discontinuation occurred in 66 (12\%) of 535 patients in the sunitinib group, most commonly fatigue (seven [1\%]), increased alanine aminotransferase (five [1\%]), diarrhoea (four [1\%]), pancreatitis (four [1\%]), and thrombocytopenia (four [1\%]). Per protocol, patients who were required to discontinue nivolumab plus ipilimumab could not continue on nivolumab monotherapy. No additional treatment-related deaths were reported since the primary analysis: eight (1\%) of 547 patients in the nivolumab plus ipilimumab group died of treatment-related causes (one each due to pneumonitis, pneumonia and aplastic anaemia, immune-mediated bronchitis, lower gastrointestinal haemorrhage, haemophagocytic syndrome, sudden death, liver toxic effects, and lung infection) and four (1\%) of 535 patients in the sunitinib group (two cardiac arrests and one each of heart failure and multiple organ failure). ${ }^{1}$ Among all treated patients, $212(39 \%)$ of 547 patients in the nivolumab plus ipilimumab died of any cause (disease 173 [32\%]; study drug toxicity eight [1\%]; unknown or other reasons 31 [6\%]), and 251 (47\%) of 535 patients in the sunitinib group died of any cause (disease 221 [41\%]; study drug toxicity four [1\%]; unknown or other reasons 26 [5\%] of 535). 


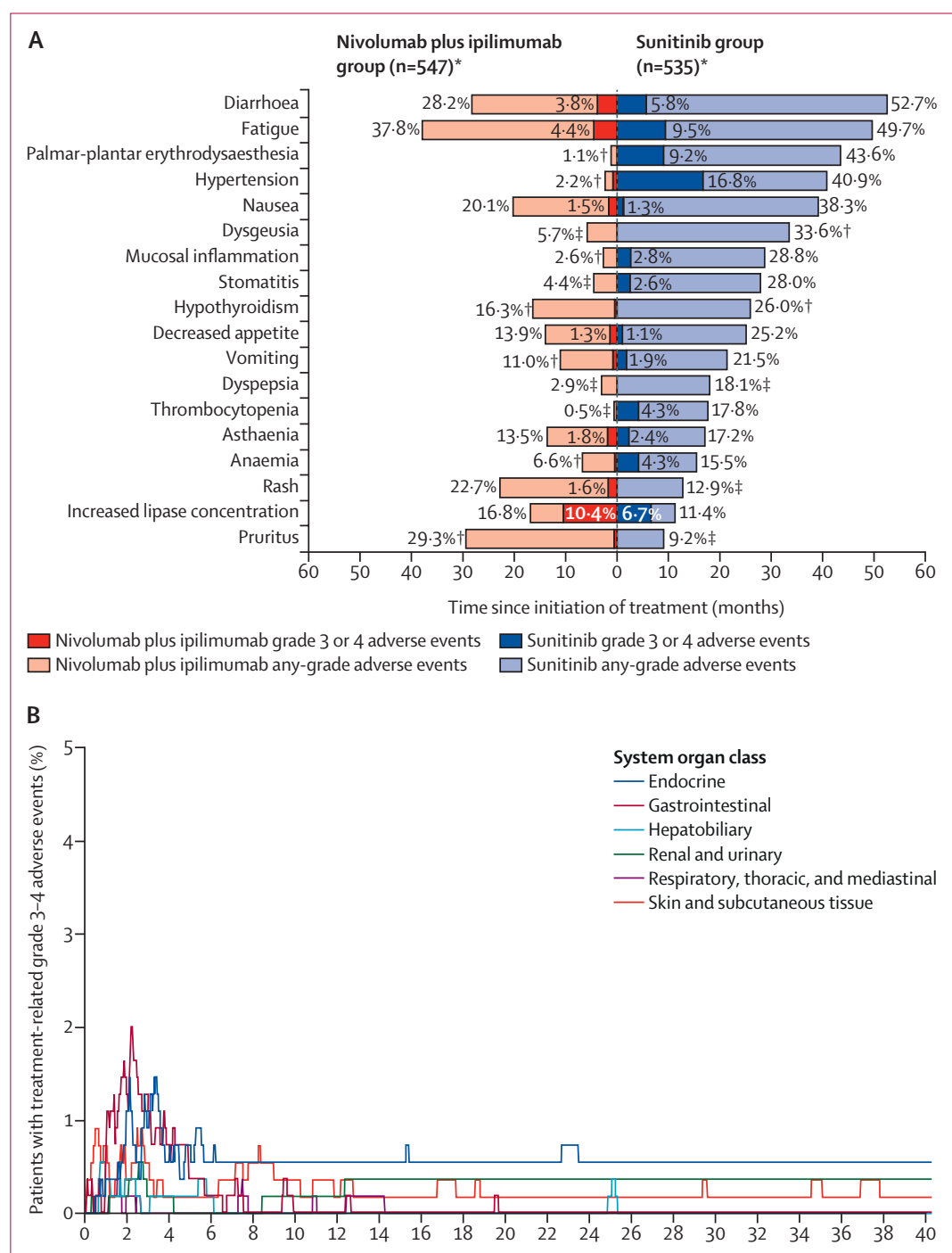

C

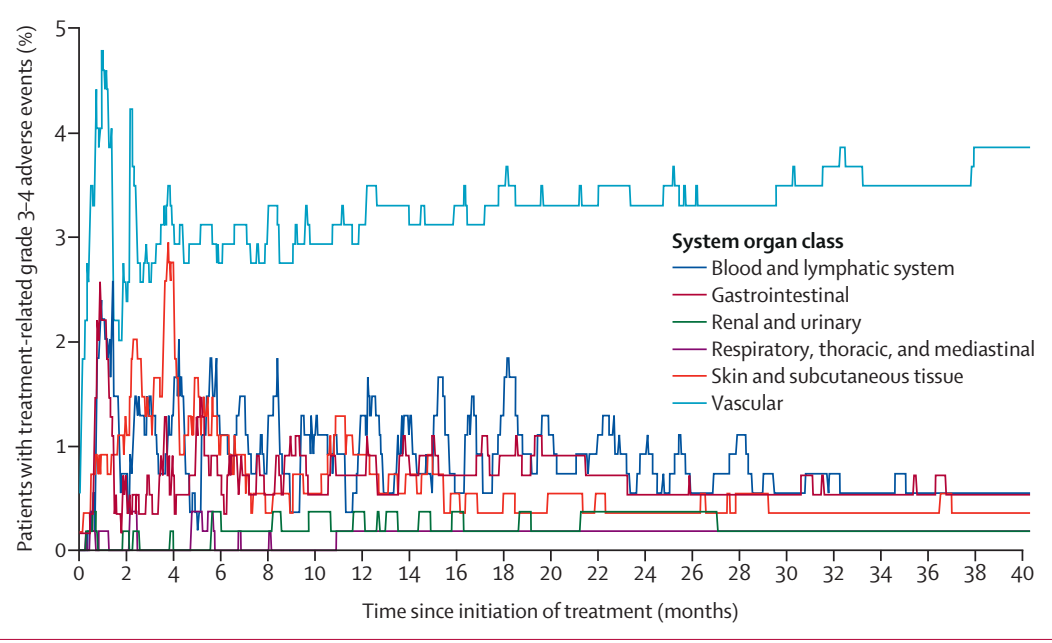

Treatment-related select adverse events (potentially immune-mediated) of any grade were reported in $443(81 \%)$ of 547 patients treated with nivolumab plus ipilimumab versus 443 (83\%) of 535 patients treated with sunitinib within 30 days of the last dose (appendix pp 12,13 ), the majority of which were grade 1 or 2 in both treatment groups (table 3 ). The same preferred terms (ie, adverse event types coded by investigators) were reported for patients in the sunitinib group, although the mechanism driving those adverse events might be different from those in the nivolumab plus ipilimumab group. Most treatment-related select adverse events with nivolumab plus ipilimumab resolved, apart from some select endocrine treatment-related adverse events (appendix pp 12, 13), which were managed with appropriate hormonal therapies. Overall, 157 (29\%) of 547 patients treated with nivolumab plus ipilimumab received $40 \mathrm{mg}$ or more of prednisone daily or equivalent to manage select treatment-related adverse events; $102(19 \%)$ patients received $40 \mathrm{mg}$ or more of prednisone daily or equivalent continuously for 2 weeks or longer, and $53(10 \%)$ received $40 \mathrm{mg}$ or more of prednisone daily or equivalent continuously for 30 days or more.

\section{Discussion}

During the extended follow-up of the CheckMate 214 trial, a significant overall survival benefit was maintained with nivolumab plus ipilimumab versus sunitinib with an early and consistent separation of the Kaplan-Meier survival curves in both the intermediate-risk poor-risk and intention-to-treat populations. The intention-to-treat group included patients with IMDC favourable, intermediate, and poor-risk disease, whereas the primary objective of the study was to assess overall survival and other efficacy endpoints in the intermediate-risk and poor-risk population. 30-month overall survival was $60 \%$ for intermediate-risk or poor-risk patients treated with nivolumab plus ipilimumab versus $47 \%$ for those treated with sunitinib (64\% vs $56 \%$ in the

Figure 5: Any-grade treatment-related adverse events occurring in more than $15 \%$ of patients in either group with treatment-related grade 3-4 adverse events (all treated patients) (A) and proportion of patients with treatment-related grade $3-4$ adverse events by common system organ class over time in the nivolumab plus ipilimumab group (B) and in the sunitinib group (C)

*Additional patients reported common any-grade treatment-related adverse events with longer follow-up compared with the primary database lock (nivolumab plus ipilimumab group: diarrhoea [n=9], pruritus [n=6], rash [n=6], fatigue [n=5], hypothyroidism [ $n=4]$, asthaenia [ $n=2]$, increased lipase [n=2], anaemia [n=2], palmar-plantar erythrodysaesthesia [n=1], nausea [n=1], mucosal inflammation $[n=1]$, stomatitis $[n=1]$, decreased appetite $[n=1]$, vomiting $[n=1]$, dyspepsia $[n=1]$, and thrombocytopenia [ $n=1]$; sunitinib group: vomiting $[n=5]$, hypothyroidism $[n=5]$, diarrhoea [ $n=4]$, increased lipase [ $n=3]$, hypertension [ $n=3]$, nausea [ $n=3]$, fatigue $[n=2]$, rash [ $n=2]$, palmar-plantar erythrodysaesthesia [ $n=2]$, mucosal inflammation [ $n=2]$, decreased appetite [ $n=2]$, asthaenia [ $n=1]$, dysgeusia [ $n=1]$, stomatitis [n=1], and dyspepsia [n=1]). $\dagger<1 \%$ reported grade $3-4$ treatment-related adverse events. $¥$ No patients reported a grade $3-4$ treatment-related adverse event. 


\begin{tabular}{|c|c|c|c|c|c|c|}
\hline & \multicolumn{3}{|c|}{ Nivolumab plus ipilimumab group $(n=547)$} & \multicolumn{3}{|c|}{ Sunitinib group $(n=535)$} \\
\hline & Grade 1-2 & Grade 3 & Grade 4 & Grade 1-2 & Grade 3 & Grade 4 \\
\hline Skin & $252(46 \%)$ & $21(4 \%)$ & 0 & $251(47 \%)$ & $53(10 \%)$ & $1(<1 \%)$ \\
\hline Pruritus & $157(29 \%)$ & $3(<1 \%)$ & 0 & $49(9 \%)$ & 0 & 0 \\
\hline Rash & $115(21 \%)$ & $9(2 \%)$ & 0 & $69(13 \%)$ & 0 & 0 \\
\hline Rash (maculo-papular) & $43(8 \%)$ & $8(1 \%)$ & 0 & $21(4 \%)$ & $1(<1 \%)$ & 0 \\
\hline Erythema & $16(3 \%)$ & 0 & 0 & $5(<1 \%)$ & 0 & 0 \\
\hline Rash (macular) & $8(1 \%)$ & 0 & 0 & $2(<1 \%)$ & 0 & 0 \\
\hline Pruritus generalised & $8(1 \%)$ & $1(<1 \%)$ & 0 & $2(<1 \%)$ & 0 & 0 \\
\hline Urticaria & $7(1 \%)$ & $1(<1 \%)$ & 0 & $2(<1 \%)$ & 0 & 0 \\
\hline Rash (pruritic) & $6(1 \%)$ & $1(<1 \%)$ & 0 & $1(<1 \%)$ & 0 & 0 \\
\hline Palmar-plantar erythrodysaesthesia & $5(<1 \%)$ & $1(<1 \%)$ & 0 & $184(34 \%)$ & $48(9 \%)$ & $1(<1 \%)$ \\
\hline Skin exfoliation & $3(<1 \%)$ & 0 & 0 & $14(3 \%)$ & 0 & 0 \\
\hline Blister & $2(<1 \%)$ & 0 & 0 & $7(1 \%)$ & $3(<1)$ & 0 \\
\hline Eczema & $2(<1 \%)$ & 0 & 0 & $6(1 \%)$ & 0 & 0 \\
\hline Skin hypopigmentation & $2(<1 \%)$ & 0 & 0 & $15(3 \%)$ & 0 & 0 \\
\hline Endocrine & $142(26 \%)$ & $31(6 \%)$ & $7(1 \%)$ & $165(31 \%)$ & $1(<1 \%)$ & 0 \\
\hline Hypothyroidism & $87(16 \%)$ & $2(<1 \%)$ & 0 & $138(26 \%)$ & $1(<1 \%)$ & 0 \\
\hline Hyperthyroidism & $57(10 \%)$ & $2(<1 \%)$ & 0 & $13(2 \%)$ & 0 & 0 \\
\hline Adrenal insufficiency & $17(3 \%)$ & $9(2 \%)$ & $2(<1 \%)$ & 0 & 0 & 0 \\
\hline Thyroiditis & $15(3 \%)$ & $1(<1 \%)$ & 0 & 0 & 0 & 0 \\
\hline $\begin{array}{l}\text { Blood thyroid stimulating hormone } \\
\text { increased }\end{array}$ & $12(2 \%)$ & 0 & 0 & $29(5 \%)$ & 0 & 0 \\
\hline Hypophysitis & $7(1 \%)$ & $13(2 \%)$ & $2(<1 \%)$ & 0 & 0 & 0 \\
\hline Diabetes & $4(<1 \%)$ & 0 & $2(<1 \%)$ & 0 & 0 & 0 \\
\hline Gastrointestinal & $135(25 \%)$ & $27(5 \%)$ & 0 & $251(47 \%)$ & $30(6 \%)$ & $1(<1 \%)$ \\
\hline Diarrhoea & $133(24 \%)$ & $21(4 \%)$ & 0 & $251(47 \%)$ & $30(6 \%)$ & $1(<1 \%)$ \\
\hline Colitis & $7(1 \%)$ & $11(2 \%)$ & 0 & $2(<1 \%)$ & 0 & 0 \\
\hline Hepatic & $56(10 \%)$ & $38(7 \%)$ & $9(2 \%)$ & $59(11 \%)$ & $20(4 \%)$ & 0 \\
\hline AST increased & $39(7 \%)$ & $17(3 \%)$ & $3(<1 \%)$ & $44(8 \%)$ & $7(1 \%)$ & 0 \\
\hline ALT increased & $33(6 \%)$ & $23(4 \%)$ & $5(<1 \%)$ & $42(8 \%)$ & $9(2 \%)$ & 0 \\
\hline Blood alkaline phosphatase increased & $16(3 \%)$ & $9(2 \%)$ & 0 & $11(2 \%)$ & $1(<1 \%)$ & 0 \\
\hline Blood bilirubin increased & $12(2 \%)$ & $1(<1 \%)$ & 0 & $15(3 \%)$ & $3(<1 \%)$ & 0 \\
\hline Transaminases increased & $9(2 \%)$ & $3(<1 \%)$ & $1(<1 \%)$ & $4(<1 \%)$ & $1(<1 \%)$ & 0 \\
\hline$\gamma$-glutamyltransferase increased & $7(1 \%)$ & $4(<1 \%)$ & $1(<1 \%)$ & $3(<1 \%)$ & $4(<1 \%)$ & 0 \\
\hline Hepatic enzyme increased & $4(<1 \%)$ & $1(<1 \%)$ & $1(<1 \%)$ & 0 & 0 & 0 \\
\hline Renal & $48(9 \%)$ & $4(<1 \%)$ & $3(<1 \%)$ & $41(8 \%)$ & $6(1 \%)$ & 0 \\
\hline Blood creatinine increased & $39(7 \%)$ & 0 & $1(<1 \%)$ & $34(6 \%)$ & $2(<1 \%)$ & 0 \\
\hline Acute kidney injury & $8(1 \%)$ & $3(<1 \%)$ & $1(<1 \%)$ & $6(1 \%)$ & $3(<1 \%)$ & 0 \\
\hline Pulmonary & $28(5 \%)$ & $6(1 \%)$ & 0 & $1(<1 \%)$ & 0 & 0 \\
\hline Pneumonitis & $26(5 \%)$ & $6(1 \%)$ & 0 & 0 & 0 & 0 \\
\hline $\begin{array}{l}\text { Data are } n(\%) \text {. Data include events of any gra } \\
\text { group. Patients could have experienced more } \\
\text { aminotransferase. }\end{array}$ & even & 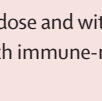 & $\begin{array}{l}\text { ays of la } \\
\text { advers }\end{array}$ & $y \cdot A L T=c$ & $\begin{array}{l}\text { ore of all } \\
\text { otransfe }\end{array}$ & $\begin{array}{l}\text { atients in ei } \\
\text { aspartate }\end{array}$ \\
\hline
\end{tabular}

intention-to-treat population). A late progression-free survival benefit was observed in both intermediate-risk or poor-risk patients and in the intention-treatpopulation, with the Kaplan-Meier curves separating after 9 and 12 months. 30-month progression-free survival was higher with nivolumab plus ipilimumab than with sunitinib in both intermediate-risk or poor-risk patients (28\% vs $12 \%)$ and in the intention-to-treat population ( $28 \%$ vs $18 \%$ ), and was consistent with nivolumab plus ipilimumab regardless of risk category. In the intention-to-treat population, in patients treated with nivolumab and ipilimumab, the median time to confirmed response was less than 3 months, and the proportion of patients achieving an objective response was higher and more patients had an ongoing response compared with the sunitinib group. These observations 
suggest that nivolumab plus ipilimumab resulted in rapid disease volume control. Notably, more responses to nivolumab plus ipilimumab were complete $(11 \%)$ and durable (88\% ongoing complete responses; $59 \%$ ongoing responses) compared with sunitinib. A high proportion of responders who discontinued nivolumab plus ipilimumab still had a treatment-free survival benefit (ie, remained progression-free or were alive) without subsequent systemic therapy, regardless of risk category.

Historically, favourable-risk patients have less aggressive disease and respond well to tyrosine kinase inhibitors, and many such patients can be managed at diagnosis with surveillance followed by delayed therapeutic intervention. ${ }^{17}$ Although the 30 -month overall survival probability in favourable-risk patients was slightly higher with sunitinib than with nivolumab and ipilimumab, survival probabilities between groups were similar. Compared with the results from the primary analysis, ${ }^{1}$ the magnitude of difference between groups regarding overall survival in the favourable-risk subgroup decreased with longer follow-up, and as median overall survival was not reached in either treatment group, the overall survival data could still be considered immature in this group. Although updated response was measured per investigator instead of per IRRC assessment, the responses with nivolumab plus ipilimumab in favourable-risk patients were durable. In this relatively small exploratory group, nine $(90 \%)$ of ten favourablerisk patients in the nivolumab plus ipilimumab group have ongoing complete response compared with only two $(40 \%)$ of five patients in the sunitinib group. Available data in favourable-risk patients suggest that further follow-up will continue to inform the benefit-risk ratio for nivolumab plus ipilimumab versus sunitinib in this group of patients.

The exploratory multivariable analysis showed that the IMDC baseline risk factors anaemia, neutrophilia, thrombocytosis, and time from diagnosis to treatment were not prognostic of overall survival with nivolumab plus ipilimumab. Interestingly, the results of the mutivariable analysis also suggest that baseline tumour PD-L1 expression of $1 \%$ or more predicts poor overall survival with sunitinib but not with nivolumab plus ipilimumab in advanced renal cell carcinoma. Neutrophil-to-lymphocyte ratio (a parameter indicating relative lymphocyte counts and marker of systemic inflammation) has shown previous prognostic value in advanced renal cell carcinoma with immune checkpoint inhibitors, and was an independent prognostic variable in both groups in the present analysis. ${ }^{18,19}$ In the current era of immune checkpoint inhibitor-based therapies, these findings highlight a need for improved prognostic models based on understanding the host response and underlying tumour biology. ${ }^{20}$

No new safety signals emerged in the nivolumab plus ipilimumab group with longer follow-up, and no treatment-related deaths occurred since the primary analysis database lock. Discontinuation of nivolumab plus ipilimumab, in part, reflected the protocol design, which specified that patients who required treatment discontinuation due to adverse events arising during nivolumab plus ipilimumab induction were not allowed to continue study treatment with nivolumab monotherapy. Interestingly, in a exploratory analysis of CheckMate $214,{ }^{21}$ similar efficacy outcomes were observed in all patients who discontinued because of treatmentrelated adverse events relative to the overall nivolumab plus ipilimumab efficacy population.

The toxicity profiles for nivolumab plus ipilimumab and sunitinib were expected to differ on the basis of disparate underlying mechanisms of action. When comparing treatment groups, common grade 3 or 4 treatmentrelated adverse events in the nivolumab plus ipilimumab group arose early and generally resolved within the first 4-6 months of treatment. By contrast, both early and chronic toxicity were apparent in the sunitinib group, despite dose adjustments. Chronic toxicities commonly observed with tyrosine kinase inhibitors, including hypertension and palmar-plantar erythrodysaesthesia, were both evident in this analysis with sunitinib and in other reports of tyrosine kinase inhibitor and immunotherapy combinations. ${ }^{22,23}$

Most treatment-related select adverse events occurring within 30 days of the last dose in the nivolumab plus ipilimumab group were low-grade, and the majority resolved and were manageable using established algorithms. However, vigilance for safety events by the health-care team throughout and after immunotherapy treatment completion is recommended. Reporting of corticosteroid use for immune checkpoint drugs has not been uniform among various studies. The initial CheckMate 214 presentation reported higher systemic corticosteroid use with nivolumab plus ipilimumab than in our current analysis; ${ }^{24}$ that figure reflected a very broad definition of any systemic corticosteroid use from allcause adverse events, and included corticosteroid use for adverse events related and unrelated to study treatment. The present analysis reported use of corticosteroids ( $\geq 40 \mathrm{mg}$ prednisone daily or equivalent) to manage treatment-related select adverse events occurring within 30 days of last dose with an appropriate denominator of all treated patients (157 [29\%] of 547 patients treated with nivolumab plus ipilimumab). This definition is more clinically meaningful than that used for the prior CheckMate 214 analyses, ${ }^{24}$ and is in line with corticosteroid-use definitions used in recent PD-L1 and VEGFR combination trials. ${ }^{22,25}$ Additionally, in a separate analysis of CheckMate 214 based on the primary database lock, health-related quality of life was maintained or significantly improved from baseline with nivolumab plus ipilimumab compared with sunitinib in intentionto-treat patients, further supporting the favourable benefit-risk profile of nivolumab plus ipilimumab versus sunitinib. ${ }^{6}$ 
Limitations should be considered when interpreting the results. Outcomes in the relatively small subset of favourable-risk patients characterised by wide $95 \%$ CIs should be considered exploratory. To this point, the favourable-risk patients were included in the intentionto-treat population along with intermediate-risk and poor-risk patients, and efficacy endpoints of objective response, progression-free survival, and overall survival were formally assessed as secondary endpoints for the trial. Additionally, the present response outcomes were analysed per investigator versus IRRC and minor differences between outcomes based on these different methods could be expected.

Ongoing work and future directions include exploring the potential role for nivolumab plus ipilimumab in treating novel patient populations including the adjuvant renal cell carcinoma setting in patients with high risk of relapse after nephrectomy (CheckMate 914, NCT03138512), and advanced renal cell carcinoma subpopulations with unmet need. A post-hoc exploratory analysis $^{26}$ of CheckMate 214 showed improved overall survival and progression-free survival benefits with nivolumab plus ipilimumab (60 patients) compared with sunitinib (52 patients) in intermediate-risk or poor-risk patients with sarcomatoid features, with a higher proportion of patients treated with nivolumab plus ipilimumab achieving an objective response $(56.7 \%$ vs $19.2 \% ; \mathrm{p}<0.0001$ ) and a higher proportion of patients achieving a complete response $(18 \cdot 3 \%$ vs $0 \%)$ compared with sunitinib; the magnitude of benefit from nivolumab plus ipilimumab compared with sunitinib was greater in these sarcomatoid-positive patients compared with the overall intermediate-risk or poor-risk population, and detailed analyses are ongoing. Furthermore, a prospective phase 3b-4 study of nivolumab plus ipilimumab in treatment-naive patients with advanced renal cell carcinoma with clear cell disease, non-clear cell disease, or non-active brain metastases (all with Karnofsky performance status $\geq 70 \%$ ), or any histology or nonactive brain metastasis with a Karnofsky performance status of 50-60\% (CheckMate 920; NCT02982954), and a phase 2 study of nivolumab plus ipilimumab versus sunitinib in patients with non-clear cell advanced renal cell carcinoma (SUNIFORECAST; NCT03075423) are ongoing. The updated CheckMate 214 results support ongoing studies combining nivolumab plus ipilimumab with novel drugs (eg, with NKTR-214 in PIVOT-02; NCT02983045). Ongoing molecular and genomic analyses of CheckMate 214 and other studies might indicate predictive biomarkers of response or prognostic factors to nivolumab plus ipilimumab. Additional CheckMate 214 analyses beyond the scope of this report, such as outcomes in patients who discontinued therapy before finishing nivolumab plus ipilimumab induction, updated healthrelated quality of life after extended follow-up, and further details of treatment-related adverse event kinetics, could be done in future investigations.
In summary, with extended follow-up, improved overall survival was maintained with nivolumab plus ipilimumab compared with sunitinib in both the intention-to-treat population and the intermediate-risk or poor-risk populations, and overall survival was similar between the treatment groups in the relatively small favourable-risk subgroup of patients in CheckMate 214. An improvement was also observed in progression-free survival with nivolumab plus ipilimumab over sunitinib in the intention-to-treat population and intermediaterisk or poor-risk patients with longer follow-up, with a notable proportion of intention-to-treat patients remaining progression-free at 30 months. Complete and durable responses were observed with nivolumab plus ipilimumab regardless of IMDC-risk based prognosis.

\section{Contributors}

RJM, SM, and NMT contributed to the conception and design of the study. All authors provided study materials or patients. MBH completed the statistical analyses. SM reviewed the clinical data. All authors contributed to the data analysis and interpretation, drafting and revising of the manuscript, and provided final approval to submit the manuscript for publication.

\section{Declaration of interests}

RJM reports grants from Bristol-Myers Squibb, during the conduct of the study; and grants and personal fees from Pfizer, Novartis, Eisai, Exelixis, and Genentech/Roche, personal fees from Merck, grants and personal fees from Eisai and Pfizer, and personal fees from Novartis, outside the submitted work. BIR reports grants and personal fees from Bristol-Myers Squibb, outside the submitted work. DFM reports personal fees from Exelixis, Array BioPharma, Bristol-Myers Squibb, Merck, Genentech/Roche, Novartis, Pfizer, and Eisai; grants from Prometheus, Bristol-Myers Squibb, Merck, Genentech/Roche, Pfizer, Exelixis, Novartis, and X4 Pharma; and personal fees from Alkermes Inc, outside the submitted work. OAF reports personal fees from Roche, Tecnofarma, Novartis, and Bristol-Myers Squibb, and other support from Pfizer, outside the submitted work. HJH reports grants and personal fees from Bristol-Myers Squibb and Merck, and personal fees from Pfizer and Exelixis, Armo Biosciences, and Novartis, during the conduct of the study. MAC reports personal fees from Astellas Pharma, AbbVie, Roche/Genentech, Pfizer, and Foundation Medicine, and grants from Bristol-Myers Squibb, AstraZeneca, Gilead Sciences, EMD Serono, and Effector, outside the submitted work. BE reports honoraria from Ipsen, Novartis, Pfizer, Bristol-Myers Squibb, Exelixis, EUSA, Aveo, and Roche. $\mathrm{BB}$ reports grants and personal fees from Pfizer and Bristol-Myers Squibb, and personal fees from Ipsen, outside the submitted work. AA reports grants, personal fees, and non-financial support from Bristol-Myers Squibb and Merck; grants and personal fees from Dynavax; and personal fees and non-financial support from Pfizer Exelixis, Bioarray, and Novartis, outside the submitted work. CP reports personal fees from Bristol-Myers Squibb, Merck Sharpe \& Dohme, Novartis, Ipsen, EUSA, Eisai, Janssen, AstraZeneca, and General Electric, and grants and personal fees from Pfizer, outside submitted work. SG reports personal fees from AstraZeneca, Exelixis and Janssen, Genentech, Sanofi/Genzyme, and EMD Serono; grants and personal fees from Bayer, Bristol-Myers Squibb, Novartis, Corvus, and Pfizer; and grants from Acceleron, Merck, Agensys, and Eisai, outside the submitted work. SB reports non-financial support from Novartis; personal fees, non-financial support, and other from Astellas and Pfizer; personal fees and non-financial support from Janssen and Merck Sharpe \& Dohme; non-financial and other support from Bristol-Myers Squibb and Roche; and non-financial support from Exelixis and AstraZeneca, outside the submitted work. SST reports clinical trial support received on behalf of his institution from Bristol-Myers Squibb and non-financial support from Bristol-Myers Squibb, during the conduct of the study; clinical trial support received on behalf of his institution from Peloton Therapeutics, Merck, Nektar Therapeutics, Calithera Biosciences, Jounce Therapeutics, Pfizer, Genentech, Prometheus Laboratories, and ARGOS 
Therapeutics; and personal fees from Calithera Biosciences and Prometheus Laboratories, outside the submitted work. PB reports advisory board funding from Bristol-Myers Squibb, Pfizer, Roche, Ipsen, Merck Sharpe \& Dohme, Janssen Cilag, and Novartis, outside the submitted work. RL-A reports honoraria from Bristol-Myers Squibb, Merck Sharpe \& Dohme, Roche, Isotopia, and Bayer; advisory role travel grant from Janssen and Pfizer; and advisory role from Sanofi and Astellas, outside the submitted work. ERP reports grants, consulting fees, and travel fees from Bristol-Myers Squibb during the conduct of the study; consultant fees from AstraZeneca, Bristol-Myers Squibb, Genentech/Roche, Merck, Novartis, Pfizer, Eli Lilly, SynerGene Therapeutics, Inovio, Clovis, Horizon Pharma, and Exelixis; funds for development of educational presentations from Bristol-Myers Squibb, Merck, Roche, and Novartis; grants to institution for conduct of clinical trials from AstraZeneca, Bristol-Myers Squibb, Merck, Peloton, Pfizer, and Astellas; and has a patent (US patent applications, numbers $14 / 588,503$ and 15/226,474). SFO reports non-financial support from Bristol-Myers Squibb, during the conduct of the study; and grants from Celldex and Novartis, outside the submitted work. BM reports personal fees and honoraria for advisory boards, speeches, and travel support from Bristol-Myers Squibb, Roche, Merck Sharpe \& Dohme,

For more on Bristol-Myers Squibb's policy on data sharing see https://www.bms.com/ researchers-and-partners/ independent-research/datasharing-request-process.html

Merck Serono, and Novartis; and personal fees from Pfizer, Ipsen, Sanofi, Astellas, Janssen, Eisai, AstraZeneca, and Amgen, outside the submitted work. TP reports grants from AstraZeneca, Roche, Novartis, Merck, Bristol-Myers Squibb, Pfizer, Roche, Ipsen, Novartis, and Exelixis, outside the submitted work. PN reports board membership, manuscript preparation, and travel fees from Bristol-Myers Squibb, outside the submitted work. SO reports grants, personal fees, and non-financial support from Bristol-Myers Squibb, Pfizer, Novartis, Eisai, and Bayer, outside the submitted work. DP reports research funding from Bristol-Myers Squibb, during the conduct of the study; personal fees and advisory board fees from Bristol-Myers Squibb, and personal fees, research funding, and advisory board funding from Pfizer, outside the submitted work. TKC reports grants, personal fees, and non-financial support from Pfizer and Exelixis, during the conduct of the study; grants and personal fees from AstraZeneca, Bayer, Bristol-Myers Squibb, Cerulean, Eisai, Foundation Medicine Inc, Exelixis, Genentech, Roche, GlaxoSmithKline, Merck, Novartis, Peloton, Pfizer, Prometheus Labs, Corvus, and Ipsen; grants from Tracon; personal fees from Alligent, Up-to-Date, NCCN, Analysis Group, Michael J Hennessy (MJH) Associates Inc (healthcare communications company and several brands), L-path, Kidney Cancer Journal, Clinical Care Options, Platform Q, Navinata Healthcare, Harborside Press, American Society of Medical Oncology, New England Journal of Medicine, and The Lancet Oncology; and grants from Calithera and Takeda, outside the submitted work. FD reports grants from Novartis, Pfizer, and Ipsen, outside the submitted work. M-OG reports treatment fees from Bristol-Myers Squibb for participation in CheckMate 214 during the conduct of this trial; grants and personal fees from Novartis and Bristol-Myers Squibb; and personal fees from Pfizer, Bayer HealthCare, Astellas, Intuitive Surgical, Sanofi Aventis, Hexal, Apogepha, Amgen, AstraZeneca, Merck Sharpe \& Dohme, Janssen Cilag, ONO Pharmaceutical, Ipsen Pharma, and Medac, outside the submitted work. HG reports personal fees from Bristol-Myers Squibb, during the conduct of the study; and personal fees from Pfizer, Astellas, Ipsen, and Roche, outside the submitted work. DYCH reports personal fees from Bristol-Myers Squibb, Pfizer, and Novartis, outside the submitted work. CKK reports personal fees from Bristol-Myers Squibb, Pfizer, Eisai, Ipsen, Roche, and AstraZeneca, outside the submitted work. MRH reports grants from Pfizer, during the conduct of the study; grants from Argos, Bristol-Myers Squibb, Exelixis, and Genentech; personal fees from Argos, Exelixis, Genentech, and Bristol-Myers Squibb; consulting fees from Argos, Exelixis, and Pfizer; and speakers bureau fees from Exelixis and Genentech, outside the submitted work. YT reports grants from Astellas, AstraZeneca, ONO Pharmaceutical, Pfizer, and Chugai, and personal fees from Astellas, Bristol-Myers Squibb, Novartis, ONO Pharmaceutical, Pfizer, and Taiho, outside the submitted work. ID reports personal fees from Bristol-Myers Squibb, Novartis, Sanofi, Bayer, Pharmacyclics, Janssen, and Merck Sharpe \& Dohme; personal fees and non-financial support from Ipsen; and grants, personal fees, and non-financial support from
Genentech/Roche and AstraZeneca, outside the submitted work. VG reports grants and personal fees from Pfizer and EUSA Pharma; grants, personal fees, and non-financial support from Bristol-Myers Squibb and Ipsen; and personal fees and non-financial support from Roche, Novartis, and Eisai, during the conduct of the study; grants, personal fees, non-financial support, and stock holdings in Merck Sharpe \& Dohme and Bristol-Myers Squibb; grants from Novartis; grants, personal fees, and stock holdings in AstraZeneca; personal fees and non-financial support from Merck Serono and PharmaMar; and personal fees from Roche, Pfizer, and Lilly, outside the submitted work. MBM reports personal fees, and employment and stock holdings in Bristol-Myers Squibb, outside the submitted work. SM reports personal fees from and employment by Bristol-Myers Squibb, outside the submitted work. NMT reports grants and personal fees from Bristol-Myers Squibb, Pfizer, Novartis, and Exelixis Inc; personal fees from Nektar Therapeutics, Oncorena, Eisai Medical Research, and ONO Pharmaceutical; and grants from Calithera Biosciences, outside the submitted work. PS, VN, and BR declare no competing interests.

\section{Data sharing}

Bristol-Myers Squibb's policy on data sharing can be found at the company's website. Deidentified and anonymised datasets of clinical trial information, including patient-level data, will be shared with external researchers for proposals that are complete, for which the scientific request is valid and the data are available, consistent with safeguarding patient privacy and informed consent. Upon execution of an agreement, the deidentified and anonymised data sets can be accessed via a secured portal that provides an environment for statistical programming with $\mathrm{R}$. The trial protocol and statistical analysis plan will also be available. Data will be available for 2 years from the study completion or termination of the programme (August, 2024).

\section{Acknowledgments}

CheckMate 214 was funded by Bristol-Myers Squibb and ONO Pharmaceutical. A data confidentiality agreement was in place between Bristol-Myers Squibb and the investigators. All authors vouch for the completeness and accuracy of the data and analyses and for the adherence of the trial to the protocol. We thank the patients and their families who are making this study possible; the late Paul Gagnier, the initial medical monitor; Jennifer McCarthy, the CheckMate 214 protocol manager; and Dako, an Agilent Technologies Inc company, for collaborative development of the PD-L1 immunohistochemistry 28-8 pharmDx assay. Professional medical writing and editorial assistance were provided by Jennifer Tyson and Lawrence Hargett of Parexel, funded by Bristol-Myers Squibb. Patients treated at the Memorial Sloan Kettering Cancer Center were supported in part by Memorial Sloan Kettering Cancer Center Support Grant (Core Grant, number P30 CA008748).

\section{References}

1 Motzer RJ, Tannir NM, McDermott DF, et al. Nivolumab plus ipilimumab versus sunitinib in advanced renal-cell carcinoma. N Engl J Med 2018; 378: 1277-90.

2 Bristol-Myers Squibb. OPDIVO (nivolumab). Princeton, NJ: Bristol-Myers Squibb, 2018.

3 Bristol-Myers Squibb. YERVOY (ipilimumab). Princeton, NJ: Bristol-Myers Squibb, 2018.

4 Jonasch E. Updates to the management of kidney cancer. J Natl Compr Canc Netw 2018; 16: 639-41.

5 European Medicines Agency. Opdivo. 2019. https://www.ema. europa.eu/en/medicines/human/EPAR/opdivo (accessed March 20, 2019).

6 Cella D, Grünwald V, Escudier B, et al. Patient-reported outcomes of patients with advanced renal cell carcinoma treated with nivolumab plus ipilimumab versus sunitinib (CheckMate 214): a randomised, phase 3 trial. Lancet Oncol 2019; 20: 297-310.

7 Anagnostou V, Yarchoan M, Hansen AR, et al. Immuno-oncology trial endpoints: capturing clinically meaningful activity. Clin Cancer Res 2017; 23: 4959-69.

8 Heng DY, Xie W, Regan MM, et al. Prognostic factors for overall survival in patients with metastatic renal cell carcinoma treated with vascular endothelial growth factor-targeted agents: results from a large, multicenter study. J Clin Oncol 2009; 27: 5794-99. 
9 McDermott DF, Rini BI, Motzer RI, et al. Treatment-free survival (TFS) after discontinuation of first-line nivolumab (NIVO) plus ipilimumab (IPI) or sunitinib (SUN) in intention-to-treat (ITT) and IMDC favorable-risk patients (pts) with advanced renal cell carcinoma (aRCC) from CheckMate 214. Proc Am Soc Clin Oncol 2019; 37 (suppl 7): 564.

10 National Cancer Institute. Common Terminology Criteria for Adverse Events (CTCAE) version 4.0. http://ctep.cancer.gov/ protocolDevelopment/electronic_applications/ctc.htm\#ctc_40 (accessed Sept 25, 2017).

11 Kaplan EL, Meier P. Nonparametric estimation from incomplete observations. J Am Stat Assoc 1958; 53: 457-81.

12 Motzer RJ, Bacik J, Murphy BA, Russo P, Mazumdar M. Interferon-alfa as a comparative treatment for clinical trials of new therapies against advanced renal cell carcinoma. J Clin Oncol 2002; 20: 289-96.

13 Clopper CJ, Pearson ES. The use of confidence or fiducial limits illustrated in the case of the binomial. Biometrika 1934; 26: 404-13.

14 DerSimonian R, Laird N. Meta-analysis in clinical trials. Control Clin Trials 1986; 7: 177-88.

15 Tannir NM, Figlin RA, Gore ME, et al. Long-term response to sunitinib treatment in metastatic renal cell carcinoma: a pooled analysis of clinical trials. Clin Genitourin Cancer 2017; 16: 30171-74.

16 Molina AM, Lin X, Korytowsky B, et al. Sunitinib objective response in metastatic renal cell carcinoma: analysis of 1059 patients treated on clinical trials. Eur J Cancer 2014; 50: 351-58.

17 Atkins MB, Tannir NM. Current and emerging therapies for first-line treatment of metastatic clear cell renal cell carcinoma. Cancer Treat Rev 2018; 70: 127-37.

18 Lalani AA, Xie W, Martini DJ, et al. Change in neutrophil-to-lymphocyte ratio (NLR) in response to immune checkpoint blockade for metastatic renal cell carcinoma. J Immunother Cancer 2018; 6: 5.

19 Zahoor H, Barata PC, Jia X, et al. Patterns, predictors and subsequent outcomes of disease progression in metastatic renal cell carcinoma patients treated with nivolumab. J Immunother Cancer 2018; 6: 107.
20 McDermott DF, Huseni MA, Atkins MB, et al. Clinical activity and molecular correlates of response to atezolizumab alone or in combination with bevacizumab versus sunitinib in renal cell carcinoma. Nat Med 2018; 24: 749-57.

21 Tannir NM, Motzer RJ, Plimack ER, et al. Outcomes in patients (pts) with advanced renal cell carcinoma (aRCC) who discontinued (DC) first-line nivolumab + ipilimumab (N+I) or sunitinib (S) due to treatment-related adverse events (TRAEs) in CheckMate 214. Proc Am Soc Clin Oncol 2019; 37 (suppl 7): 581.

22 Motzer RJ, Penkov K, Haanen J, et al. Avelumab plus axitinib versus sunitinib for advanced renal-cell carcinoma. N Engl J Med 2019; 380: 1103-15

23 Rini BI, Plimack ER, Stus V, et al. Pembrolizumab plus axitinib versus sunitinib for advanced renal-cell carcinoma. $N$ Engl J Med 2019; 380: 1116-27.

24 Escudier B, Tannir NM, McDermott DF, et al. CheckMate 214: efficacy and safety of nivolumab plus ipilimumab vs sunitinib for treatment-naïve advanced or metastatic renal cell carcinoma, including IMDC risk and PD-L1 expression subgroups. European Society of Medical Oncology 2017; Madrid, Spain; Sept 8-12, 2017. LBA5.

25 Rini BI, Atkins MB, Escudier B, et al. Atezolizumab plus bevacizumab versus sunitinib in patients with previously untreated metastatic renal cell carcinoma (IMmotion151): a multicentre, open-label, phase 3, randomised controlled trial. Lancet 2019; 393: 2404-15

26 McDermott DF, Motzer RJ, Rini BI, et al. CheckMate 214 post-hoc analyses of nivolumab plus ipilimumab or sunitinib in IMDC intermediate/poor-risk patients with previously untreated advanced renal cell carcinoma with sarcomatoid features. Proc Am Soc Clin Oncol 2019; 37 (suppl 15): 4513. 